\title{
Interferon-dependent IL-10 production by Tregs limits tumor Th17 inflammation
}

\author{
C. Andrew Stewart, ${ }^{1}$ Hannah Metheny, ${ }^{1}$ Noriho lida, ${ }^{1}$ Loretta Smith, ${ }^{1}$ \\ Miranda Hanson, ${ }^{1}$ Folkert Steinhagen, ${ }^{1,2}$ Robert M. Leighty, ${ }^{3}$ Axel Roers, ${ }^{4}$ \\ Christopher L. Karp, ${ }^{5}$ Werner Müller, ${ }^{6}$ and Giorgio Trinchieri ${ }^{1}$
}

\begin{abstract}
${ }^{1}$ Cancer and Inflammation Program, Center for Cancer Research, National Cancer Institute, NIH, Frederick, Maryland, USA.
2Department for Anesthesiology and Intensive Care Medicine, University Hospital Bonn, Bonn, Germany. ${ }^{3}$ Data Management Services Inc., Frederick National Laboratory for Cancer Research, Frederick, Maryland, USA. ${ }^{4}$ Medical Faculty Carl Gustav Carus, University of Technology Dresden, Dresden, Germany. ${ }^{5}$ Division of Molecular Immunology, Cincinnati Children's Hospital Research Foundation, Cincinnati, Ohio, USA.

${ }^{6 B i l l}$ Ford Chair of Cellular Immunology, Faculty of Life Sciences, University of Manchester, Manchester, United Kingdom.
\end{abstract}

\begin{abstract}
The capacity of IL-10 and Tregs in the inflammatory tumor microenvironment to impair anticancer Th1 immunity makes them attractive targets for cancer immunotherapy. IL-10 and Tregs also suppress Th17 activity, which is associated with poor prognosis in several cancers. However, previous studies have overlooked their potential contribution to the regulation of pathogenic cancer-associated inflammation. In this study, we investigated the origin and function of IL-10-producing cells in the tumor microenvironment using transplantable tumor models in mice. The majority of tumor-associated IL-10 was produced by an activated Treg population. IL-10 production by Tregs was required to restrain Th17-type inflammation. Accumulation of activated IL-10 ${ }^{+}$ Tregs in the tumor required type I IFN signaling but not inflammatory signaling pathways that depend on TLR adapter protein MyD88 or IL-12 family cytokines. IL-10 production limited Th17 cell numbers in both spleen and tumor. However, type I IFN was required to limit Th17 cells specifically in the tumor microenvironment, reflecting selective control of tumor-associated Tregs by type I IFN. Thus, the interplay of type I IFN, Tregs, and IL-10 is required to negatively regulate Th17 inflammation in the tumor microenvironment. Therapeutic interference of this network could therefore have the undesirable consequence of promoting Th17 inflammation and cancer growth.
\end{abstract}

\section{Introduction}

Cancer establishes an inflammatory tumor microenvironment (TME) that provides growth factors for tumor and stromal cells, promotes angiogenesis, and limits antitumor immune responses $(1,2)$. In many diverse cancers, the TME exhibits a bias toward Th17-type inflammation, high numbers of immunosuppressive cells including Tregs, and expression of IL-10 and other antiinflammatory cytokines (1-3). The goal of cancer immunotherapy is to repolarize the TME to promote effective Th1- and cytotoxic $\mathrm{T}$ cell-mediated antitumor immunity. Obstacles to this, such as Tregs and IL-10, are therefore important therapeutic targets. However, the consequences of their targeting on baseline TME inflammation and hence the potential effects on tumor progression are poorly understood.

$\mathrm{CD}^{+}$Tregs are critical in maintaining central tolerance, preventing autoimmunity, and limiting the extent of inflammation $(4,5)$. Canonical Tregs are defined by expression of the FoxP3 transcription factor whose absence leads to a fatal T cell-mediated lymphoproliferative and autoimmune disorder in mice and humans $(6,7)$. Through a variety of effector mechanisms, Tregs control inflammation and immunity in multiple contexts, including the regulation of Th1-, Th2-, and Th17-type inflammatory responses (8-11). In preclinical models of both prophylactic and therapeutic cancer therapy, Tregs limit the generation of Th1 responses that drive $\mathrm{CD}^{+} \mathrm{T}$ cells and IFN- $\gamma$-dependent antitumor immunity (12-16). Furthermore, the mechanism of action of the humanized anti-CTLA-4 monoclonal antibody ipilimumab (Bristol Myers

Conflict of interest: The authors have declared that no conflict of interest exists. Citation for this article: J Clin Invest. 2013;123(11):4859-4874. doi:10.1172/JCI65180.
Squibb), which was given US FDA approval in 2011 (17) for treatment of advanced melanoma, depends on its ability to block Tregs and release APCs from Treg inhibition. Therefore, as Tregs inhibit Th1-type antitumor responses in these situations, blockade of their activity can provide effective therapy against cancer.

IL-10 is a cytokine with broad antiinflammatory properties. It acts primarily on APCs, including dendritic cells, monocytes, and macrophages, by inhibiting production of proinflammatory cytokines such as TNF and IL-12 and blocking cell maturation and upregulation of costimulatory molecules (18-22). This potent inhibitory effect on APCs makes the blockade of IL-10 a potential strategy for cancer therapy. Indeed, stimulation of tumor-resident APCs with Toll-like receptor agonists leads to poor responses unless IL-10 signaling is blocked through targeting of IL-10, IL-10R, or STAT3. The blockade leads to increased proinflammatory cytokine production, tumor necrosis, upregulation of costimulatory CD40, migration of dendritic cells to draining lymph nodes, and induction of antitumor inflammation and immunity (23-27). Therefore, IL-10 could be an important therapeutic target when combined with other immunotherapy.

However, the natural role of IL-10 in the TME of progressing tumors is complex, and it is not clear that blockade of IL-10 can promote Th1 antitumor inflammation in the absence of other therapeutic interventions. In addition to its antiinflammatory function, IL-10 has been shown to activate innate and adaptive immunity by promoting proliferation and activation of subsets of $\mathrm{CD}^{+} \mathrm{T}$ cells, NK cells, and B cells and inducing receptors involved in immune-complex recognition and phagocytosis on monocytes $(20,28,29)$. Indeed, IL-10 plays a protective anticancer role in some contexts by promoting cytotoxic T cell activity and IFN- $\gamma$ 
A

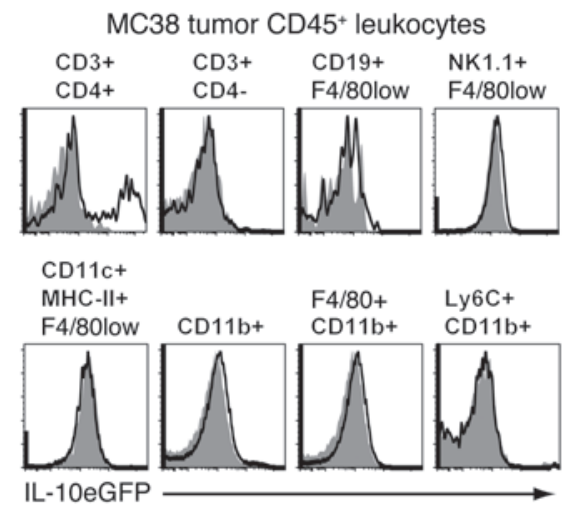

D

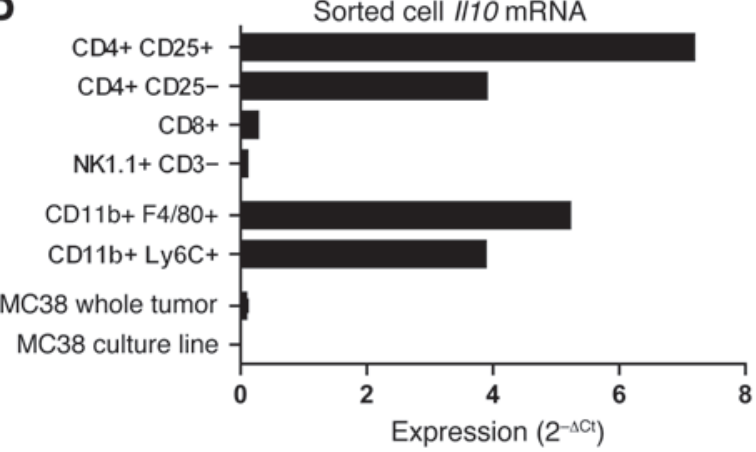

B

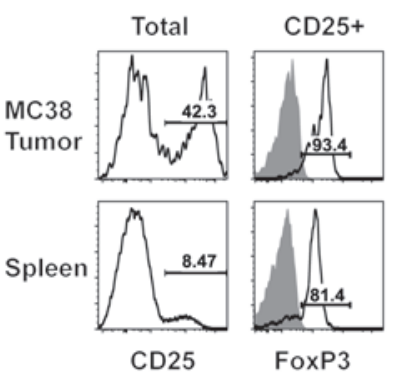

C
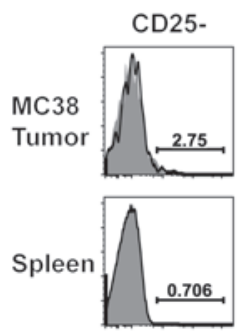

IL-10eGFP
$\mathrm{CD}^{+}{ }^{+} \mathrm{T}$ cells
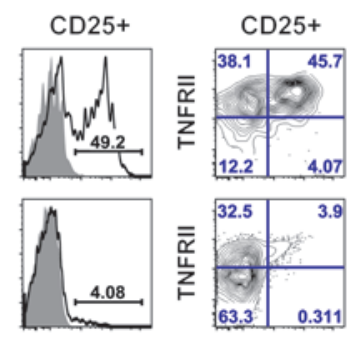

E

Tumor $\| 10$ mRNA

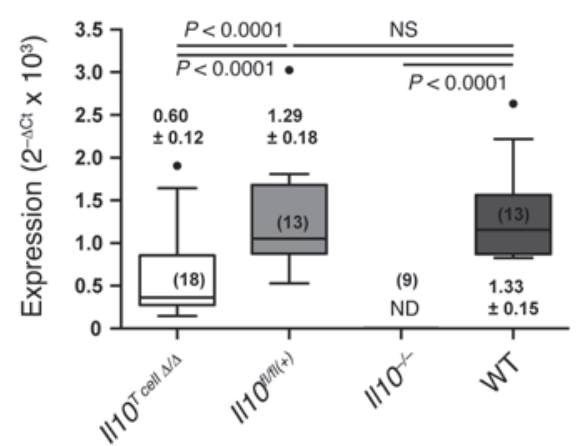

\section{Figure 1}

Tumor Tregs are a predominant source of /110. (A and C) Expression of IL-10eGFP in leukocyte populations (A) and CD4+ T cells (C) from MC38 tumor grown in VERT-X mice (black line) or B6 WT (gray) mice. Statistics show percentage of cells in each quadrant for 1 representative experiment of 3 or more. (B) Definition of tumor CD4+ Tregs by intracellular staining of FoxP3 (black line) or isotype (gray) staining. Percentage of gated cells is shown. (D) Real-time quantitative PCR for I/10 on sorted cell populations from MC38 tumor, normalized to Hprt. Experiment is representative of 3. (E) I/10 expression in MC38 tumor from T cell-conditional I/10-knockout mice (I/10 cell/L$)$. RNA was extracted from unprocessed MC38 tumors from indicated strains of mice and real-time quantitative PCR performed for I/10. Relative expression of $/ / 10$ to Hprt is shown in a boxand-whiskers plot (ND, not detected). Data were combined from 2 independent experiments and statistical analysis performed by mixed-effects ANOVA (Tukey-Kramer). Mean \pm SEM and $n$ are also shown.

production and preventing microbiota-dependent enterocolitis and colitis-associated cancer (30-33). Therefore, this study aims to shed light on IL-10's role in carcinogenesis, with the ultimate goal of focusing on the strategy for therapeutic intervention.

Th17 cells produce IL-17A, IL-17F, and IL-22, which promote antibacterial, antifungal and wound-healing inflammation, but may otherwise induce a protumor environment (34, 35). IL-17A promotes angiogenesis and lymphangiogenesis by inducing factors such as VEGF by tumor and stromal cells $(34,36)$. Furthermore, IL-17A stimulates proliferation and recruitment of neutrophils (37) that are critical mediators of angiogenesis, promote tissue remodeling, and can promote DNA damage through production of radical oxygen species (38). Contradictory experimental evidence suggests that Th17 cells can perform antitumor activity, but this activity is strictly associated with the production of IFN- $\gamma$, a Th1 cytokine, by Th17 cells $(39,40)$. However, Th17 cells are found at elevated frequencies in many human cancers and their presence or expression of IL-17A is associated with poor prognosis in hepatocellular carcinoma (HCC), colorectal cancer, breast cancer, and pancreatic cancer $(34,35)$. Therefore, factors that inhibit Th17 inflammation may protect from cancer development in this context. As Tregs and IL-10 suppress Th17 inflammation $(8,41,42)$, their activity in pre- or posttreatment cancer is a critical consideration when deciding on an intervention strategy.

A solid understanding of IL-10 and Treg biology in the TME is imperative when considering them as therapeutic cancer targets. In this study, we test the hypothesis that IL-10 regulates the inflammatory TME of progressing tumors using subcutaneous model tumor systems in C57BL/6 (B6) WT and gene-knockout mice. We identify Tregs as a predominant source of IL-10 in the TME and show that both the tumor-associated and splenic Th17 responses are restrained by IL-10 from T cells. We further show that the type I IFN signaling pathway, including Ifnar1, Stat1, and Stat2, is required for accumulation and activation of tumor Tregs and that, together, IL-10 and type I IFN act in a network that is required to limit Th17-type inflammation specifically in the TME.

\section{Results}

Tregs are the predominant source of IL-10 in the TME. Though IL-10 has been implicated in regulation of inflammation and immunity in the $\operatorname{TME}(26,31,32)$, the source and the stimuli driving its production have not yet been defined. To determine the cellular source of IL-10, we used VERT-X IL-10eGFP reporter mice encoding 
Table 1

Percentages of CD4+CD25+ Tregs and IL-10eGFP+ Tregs in VERT-X mice

\begin{tabular}{|c|c|c|c|}
\hline Organ & CD25+ of CD4 (\%) & IL-10eGFP+ of CD4+CD25- (\%) & IL-10eGFP+ of CD4+CD25+ (\%) \\
\hline MC38 tumor & $45.6 \pm 3.3$ & $7.1 \pm 2.1$ & $51.9 \pm 13.0$ \\
\hline Spleen (tumor bearing) & $6.8 \pm 1.7$ & $0.7 \pm 0.3$ & $3.3 \pm 1.1$ \\
\hline Lymph node (tumor draining) & $10.0 \pm 0.5$ & $0.3 \pm 0.1$ & $3.6 \pm 0.4$ \\
\hline Lymph node (non-tumor draining) & 10.3 & 0.1 & 4.6 \\
\hline Spleen (tumor free) & $7.1 \pm 0.5$ & $0.8 \pm 0.5$ & $3.3 \pm 0.4$ \\
\hline Lymph node (tumor free) & $10.5 \pm 1.9$ & $0.2 \pm 0.1$ & $4.6 \pm 2.0$ \\
\hline
\end{tabular}

Statistics show percentage \pm SD for $3 / 4$ VERT-X mice (except non-tumor-draining lymph node where pool of 4 mice was analyzed). All numbers indicate percentages (mean $\pm S D$ ). Data are from 1 representative experiment of 3 .

enhanced GFP (EGFP) in the 3' UTR of Il10 (43). Mice were inoculated with syngeneic MC38 tumor cells, and the expression of GFP was determined in tumor-derived cells (Figure 1A). IL-10eGFP was highly expressed in a subpopulation of CD4 $4^{+} \mathrm{T}$ cells. Other leukocyte populations showed no GFP signal, with the exception of a small shift in $\mathrm{CD}_{11} \mathrm{~b}^{+}$cells that included small numbers of neutrophils and mast cells, but predominantly corresponded with monocyte-derived cells and $\mathrm{CD} 11 \mathrm{~b}^{+} \mathrm{F} 4 / 80^{+}$macrophages in MC38 tumors. CD25 expression identified $\mathrm{CD} 4^{+} \mathrm{FoxP} 3^{+}$Tregs in the TME (Figure 1B), facilitating its use as a surface Treg marker in this context. Approximately half of the $\mathrm{CD} 25^{+}$tumor Tregs, but only approximately $7 \%$ of $\mathrm{CD}^{2} 5^{-} \mathrm{CD} 4^{+}$cells, expressed IL-10eGFP (Figure 1C and Table 1). Interestingly, in MC38 tumors, the majority of Tregs also coexpressed TNFRI, a marker of activated potent suppressor FoxP3 ${ }^{+}$Tregs (44), and almost all IL-10eGFP ${ }^{+}$Tregs were $\mathrm{TNFRII}^{+}$(Figure 1C). In the spleen, only a small proportion of CD $4^{+} \mathrm{CD} 25^{+}$Tregs expressed IL-10eGFP, but similarly to tumor Tregs, all of these coexpressed TNFRII. Most other organs of specific pathogen-free mice had low proportions of Tregs, and less than $15 \%$ of them expressed IL-10eGFP, with the exception of intestine-associated tissues in which higher frequencies of IL- $10^{+}$
Tregs were observed (Supplemental Figure 1; supplemental material available online with this article; doi:10.1172/JCI65180DS1), consistent with previous reports (45-48). To confirm that native Il10 is expressed by tumor-associated Tregs from WT mice, we performed real-time quantitative PCR on FACS-sorted populations of tumor-derived cells (Figure 1D). $\mathrm{CD} 4^{+} \mathrm{CD} 25^{+}$Tregs expressed the highest levels of Il10 mRNA compared with other $\mathrm{CD}^{+}$cells, $\mathrm{CD}^{+} \mathrm{T}$ cells, and NK cells. CD $11 \mathrm{~b}^{+} \mathrm{F} 4 / 80^{+}$macrophages and $\mathrm{CD} 11 \mathrm{~b}^{+} \mathrm{F} 4 / 80^{\text {lo }}$ myeloid-derived cells also expressed Il10 mRNA, consistent with the low level IL-10eGFP reporter expression in these populations (Figure 1A). Furthermore, only minimal levels of Il10 were detected in MC38 tumor cells ex vivo, and no expression was observed in the cultured cell line, indicating that the carcinoma cells themselves do not express $I l 10$ in either environment. To clarify the relative contribution of Tregs and myeloid cells to total tumor IL-10 and to control for an observed upregulation in Il10 expression during the mechanical disruption of the tumor required for isolation and sorting of single cells (data not shown), we analyzed Il10 mRNA expression in whole tumors from T cellconditional IL-10-knockout mice (Il10 ${ }^{T}$ cell $\Delta / \Delta$ mice). Il10 mRNA was not detected in tumors from $\mathrm{Il}_{10^{-/-}}$mice, confirming the lack
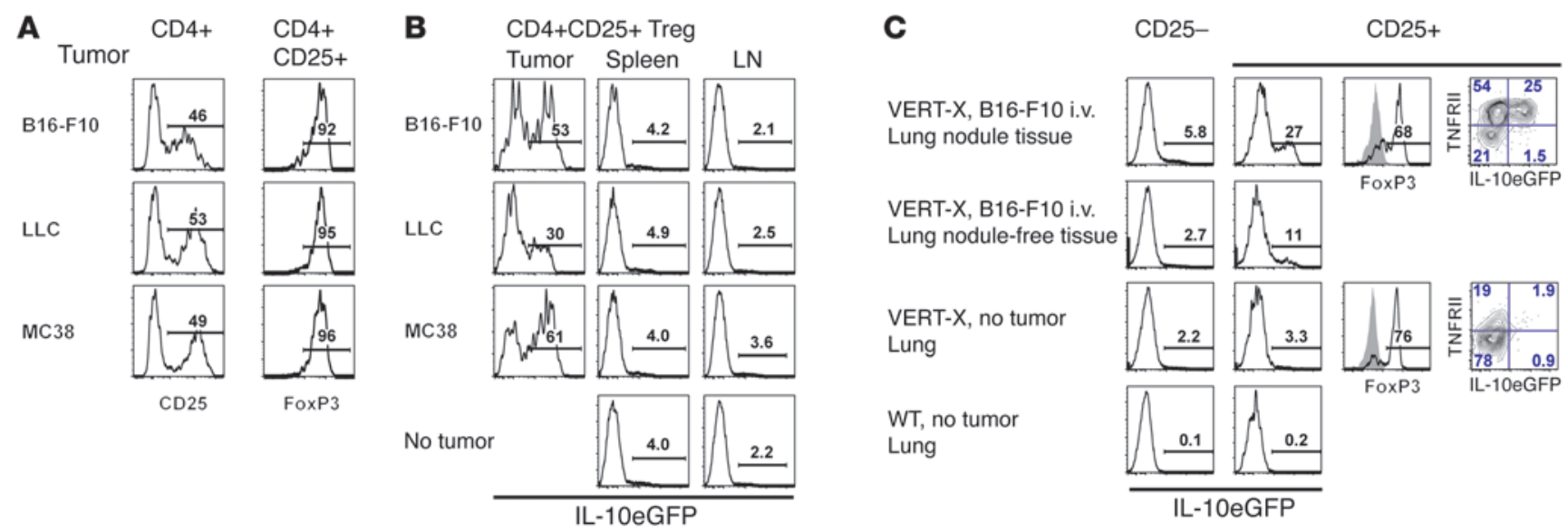

\section{Figure 2}

Expression of IL-10eGFP by CD4+CD25+ Tregs in tumor-associated tissue. Expression of IL-10eGFP was analyzed in VERT-X mice with B16-F10 melanoma, Lewis lung carcinoma (LLC), or MC38 colon carcinoma in subcutaneous (A and B) or lung-associated (C) tissue by flow cytometry. Numbers indicate percentages of positive cells in the associated gate. FoxP3 (A) and IL-10eGFP (B) staining are indicated for subcutaneous tumor tissue, spleen, and tumor-draining (inguinal) lymph node. Results are representative of 2 experiments. (C) Lungs from mice with B16-F10 tumor nodules were dissected into tumor-associated and non-tumor-associated tissue, followed by processing, staining, and flow cytometry. Data show 1 tumor-bearing mouse of 2 analyzed (individually displaying $27 \%$ or $16 \%$ IL-10eGFP+ cells in the CD $4^{+}$CD25 $5^{+}$Treg population of B16-F10 nodule-associated tissue). 
A
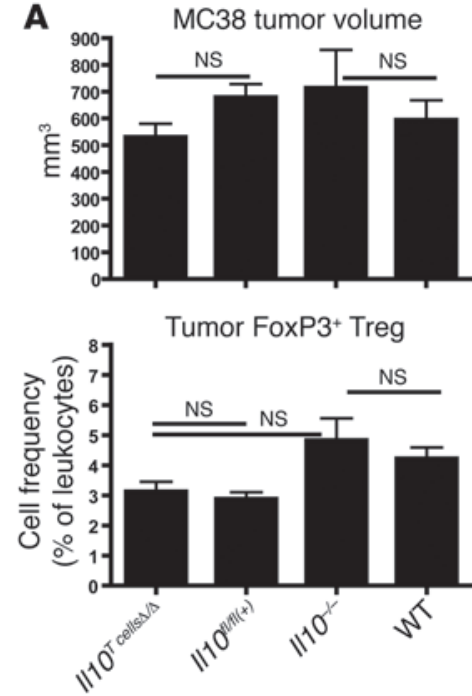

B
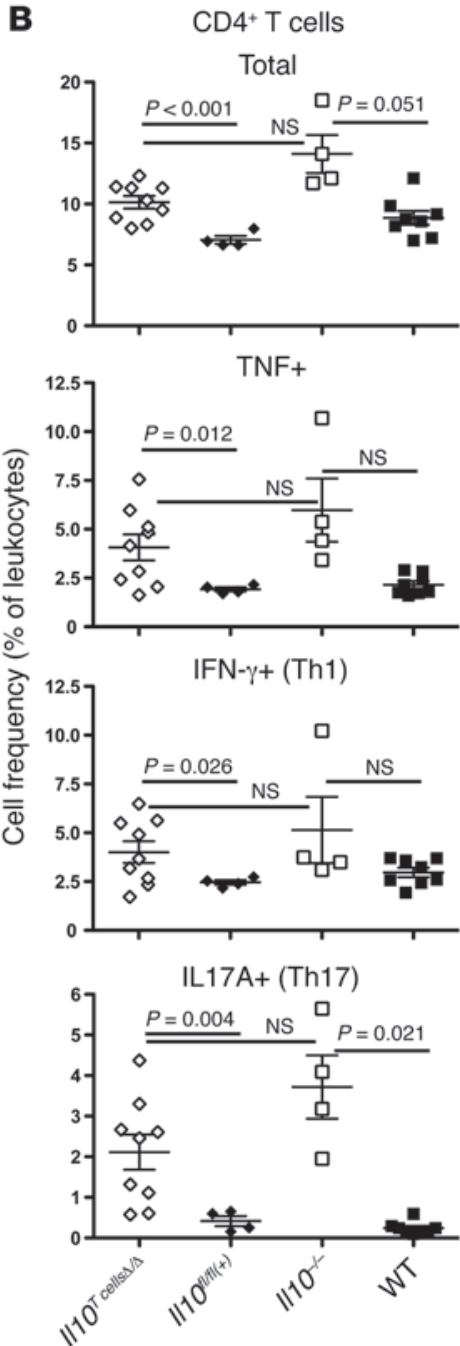

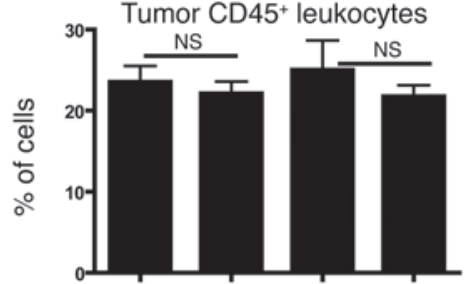

IL10+FoxP3 + Treg

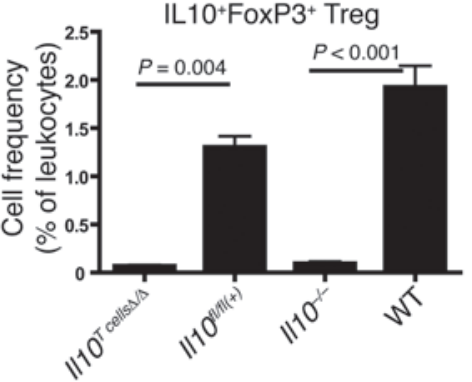

$\mathrm{CD}^{+} \mathrm{T}$ cells

Total

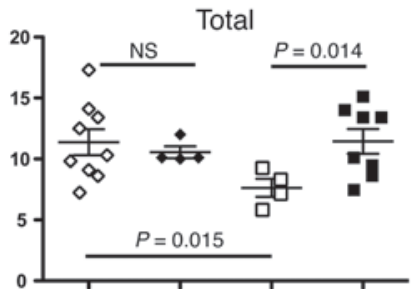

$\mathrm{TNF}+$

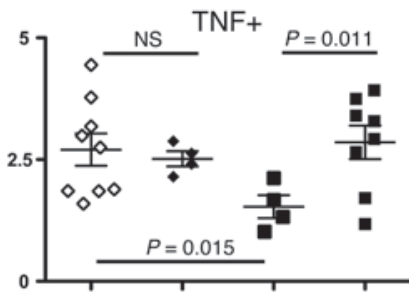

$\mathrm{IFN}-\gamma+(\mathrm{Tc} 1)$

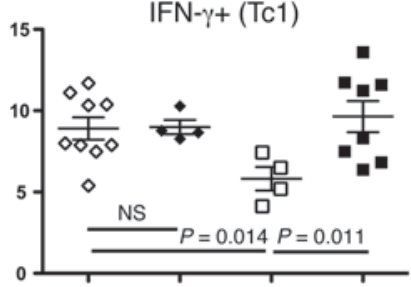

IL17A+ (Tc17)

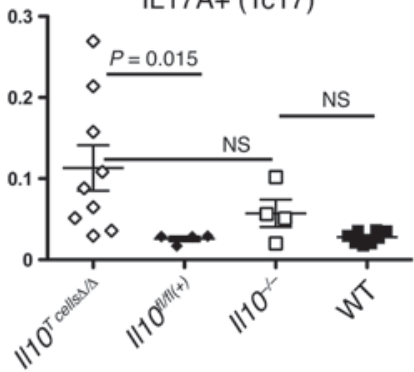

C $\mathrm{CD}^{+} \mathrm{T}$ cells

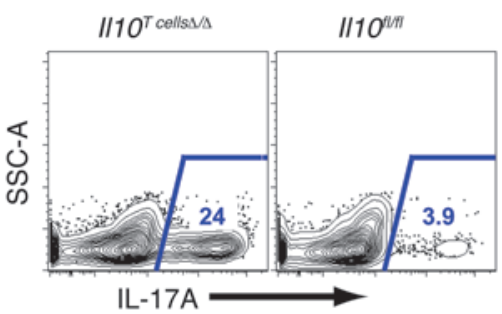

D

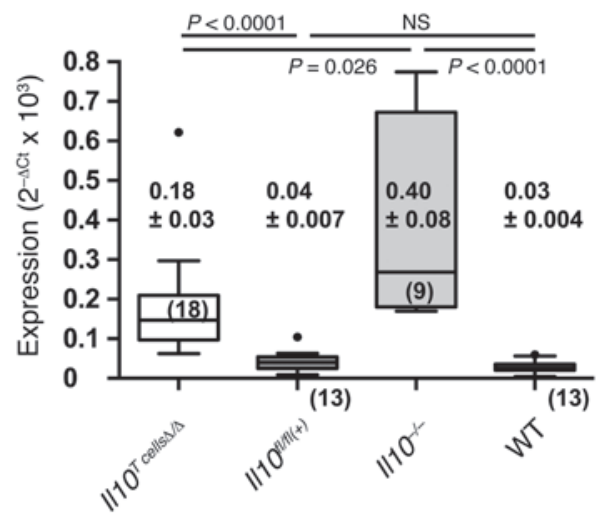

Figure 3

Expression of I/17a and frequencies of IL-17-producing cells in MC38 tumor from T cell-conditional I/10-knockout mice. (A) Tumor mass, leukocyte frequency, and FoxP3+ Treg frequencies are similar between WT and $/ 110^{-/-}$mice. (B) Frequencies of $\mathrm{CD}^{+}$and $\mathrm{CD} 8^{+} \mathrm{T}$ cells, and frequencies of IL-17, TNF, or IFN- $\gamma$-producing cells following PMA and ionomycin stimulation of MC38 tumor cells from T cell-conditional I/10-knockout mice $\left(I / 10^{T} \mathrm{cell} / \Delta\right)$, littermates $\left(/ / 10^{f / f}\right), I / 10^{-1-}$ mice, or WT mice. Cell frequencies as percentage of leukocytes and mean \pm SEM are shown with statistics from 2-tailed Student's $t$ test with Welch's correction. (C) Representative contour plot of tumor CD $45^{+}$CD $4+T$ cells from FACS analysis used to identify IL-17A+ Th17 cells as quantitated in $\mathbf{B}$. Percentages of gated cells are given. (D) Real-time quantitative PCR for $/ 117 a$ on whole MC38 tumor tissue from indicated strains of mice. Box-and-whiskers plot is shown with mean \pm SEM, $n$, and $P$ values for mixed-effects ANOVA on combined data from 2 independent experiments (each showing significance). of expression by MC38 carcinoma cells in vivo. Expression of Il10 in MC38 tumors from $I l 10^{T}$ cell $\Delta / \Delta$ mice was less than half that in tumors from littermate $\left(I l 10^{f / f l}\right)$ or WT controls $(P<0.0001)$, confirming that $\mathrm{T}$ cells are the predominant source of $I l 10$ (Figure 1E).
To extend this observation in other tumors, VERT-X mice were inoculated subcutaneously with B16-F10 melanoma, Lewis lung carcinoma, or MC38 colon carcinoma. IL-10eGFP expression was observed in $30 \%$ to $60 \%$ of $\mathrm{CD} 4{ }^{+} \mathrm{CD} 25^{+}$Tregs in each tumor 
A

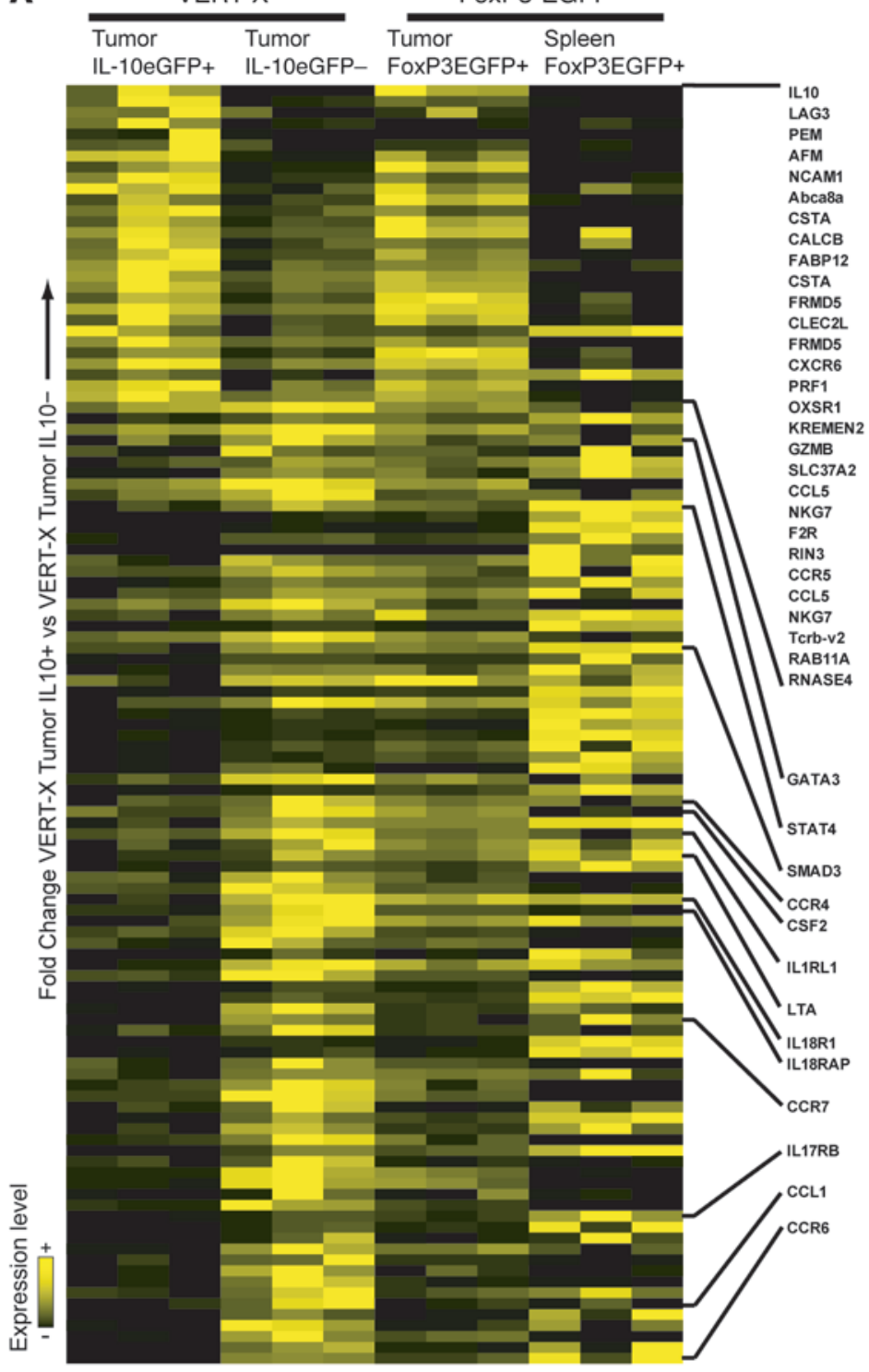

B
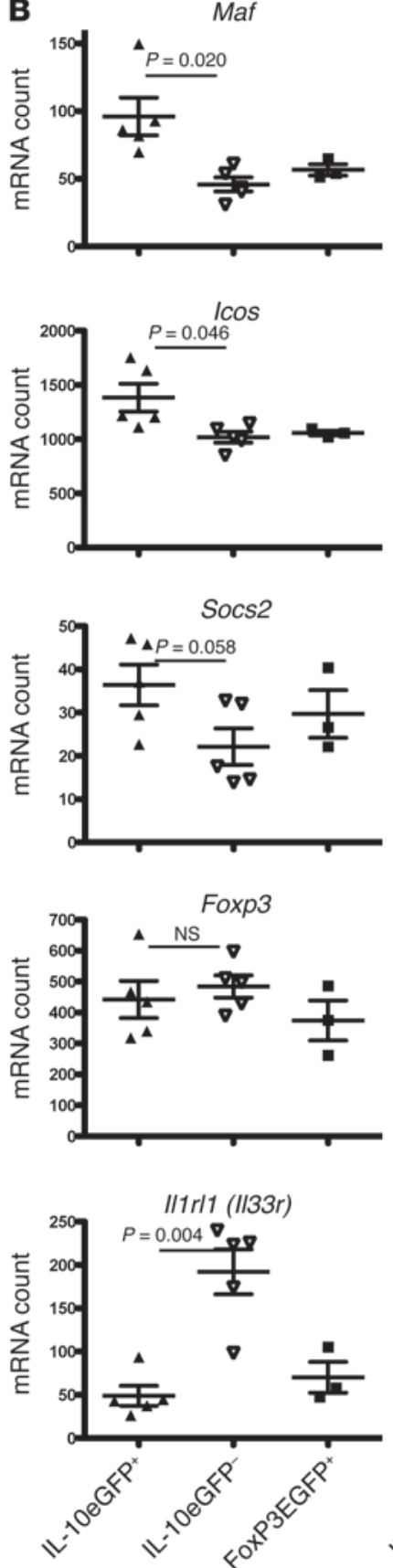

Ccr6
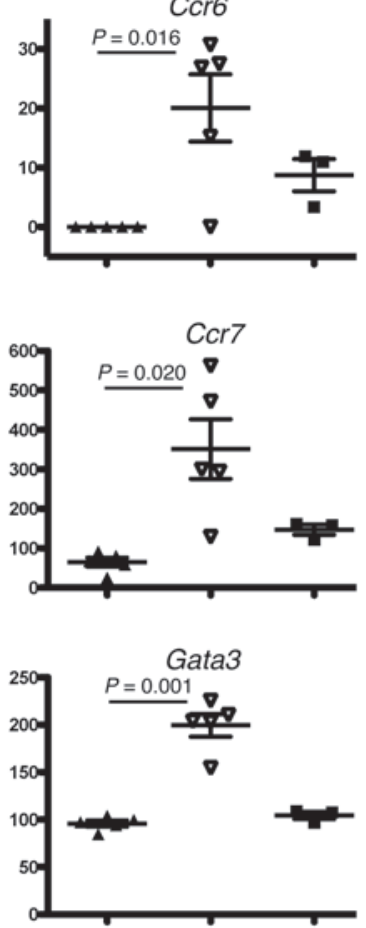

Lta
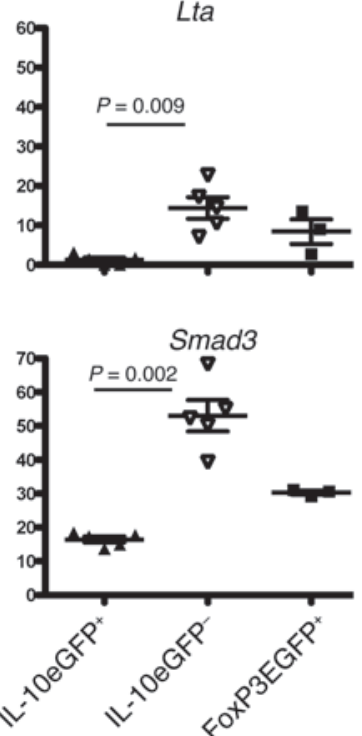

Figure 4

Expression of IL-10 by Tregs is associated with a T cell activation signature. (A) Heat map showing genes with significant differences in expression between IL-10eGFP+ and IL-10eGFP- Tregs from MC38 tumor in VERT-X mice. Genes are ranked according to fold change between IL-10eGFP+ and IL-10eGFP- Tregs and expression level indicated by color. Expression of genes by CD4+FoxP3EGFP+ Tregs from MC38 tumor and tumor-free spleen in FoxP3EGFP mice is shown for comparison. (B) nCounter analysis (Nanostring) of gene expression on independent flow-sorted samples of Tregs from MC38 tumor in VERT-X and FoxP3-EGFP mice. Each point derives from a pool of tumors in an independent experiment. Graphs show mean \pm SEM with $P$ values from 2-tailed Student's $t$ test with Welch's correction except for Ccr6 (Mann-Whitney).

type (Figure 2, A and B). Similar to MC38 carcinoma, Il10 mRNA expression in B16-F10 melanoma was all derived from host cells and approximately $50 \%$ was lost in $I l 10^{T \text { cell } \Delta / \Delta}$ mice, suggesting that Tregs were the major IL-10 producer cell subset in the TME (Supplemental Figure 2). In a nonsubcutaneous environment, lung nodules from mice that were inoculated with B16-F10 via intrave- nous administration displayed higher frequencies of IL-10eGFP ${ }^{+}$ Tregs than surrounding nodule-free tissue and higher still than normal lung Tregs (Figure 2C). Most of the Tregs in lung nodules, but not in tumor-free lungs, also expressed TNFRII. Although contamination of the metastasis-surrounding tissue by smaller B16-F10 nodules cannot be excluded, the higher frequency of 
A

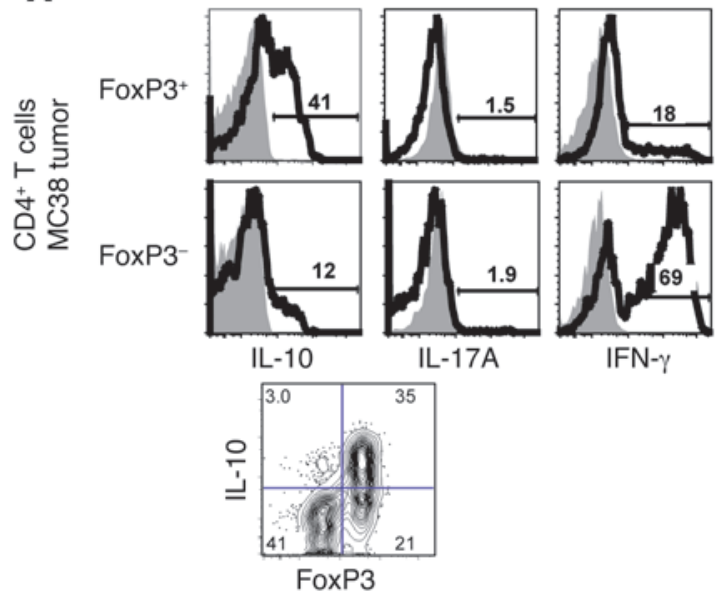

C

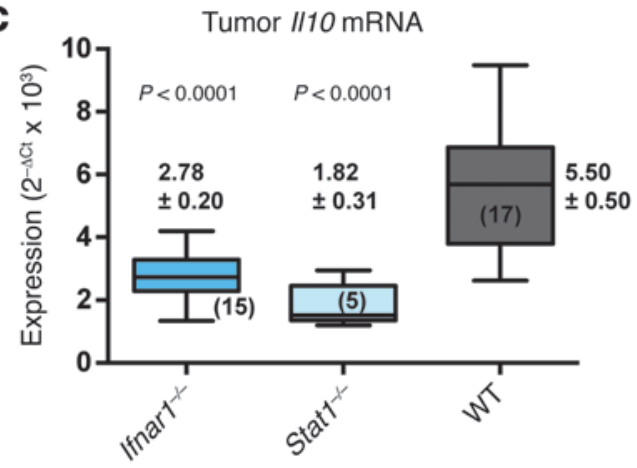

B
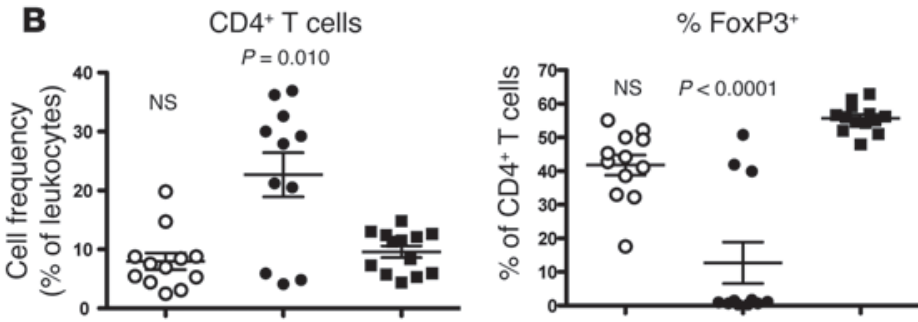

$\mathrm{IL}-10^{+} \mathrm{FoxP}^{+} \mathrm{CD}^{+} \mathrm{T}$ cells
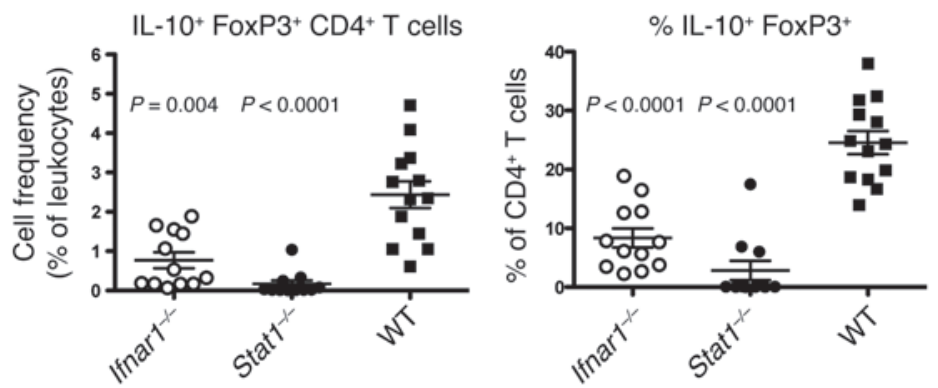

\section{Figure 5}

Ifnar1 and Stat1 are required for expression of IL-10 and Stat1. (A) Strategy for identification of CD4+ T cell subsets. Plots show intracellular staining of CD4, FoxP3, and either IL-10, IL-17A, or IFN- $\gamma$ on single-cell suspensions of MC38 tumors following stimulation with PMA and ionomycin. Percentages of gated cells are given. (B) Frequencies of IL-10+FoxP3 ${ }^{+} \mathrm{CD} 4^{+} \mathrm{T}$ cells, FoxP3 ${ }^{+} \mathrm{CD} 4^{+} \mathrm{T}$ cells, and $\mathrm{CD} 4^{+} \mathrm{T}$ cells in MC38 tumor are given as percentage of CD4 ${ }^{+}$T cells or percentage of leukocytes from Ifnar $1^{-1-}$, Stat $11^{-1-}$, and WT mice. Mean \pm SEM and $P$ values compared with WT are shown for 5 independent experiments combined. (C) Real-time quantitative PCR for II10 on whole MC38 tumor from Ifnar $1^{-1-}$, Stat1 ${ }^{-1-}$, and WT mice, normalized to Ptprc (CD45). Box-and-whiskers plots with mean $\pm \mathrm{SEM}, n$, and $P$ values compared with WT using combined data from 4 (Ifnar $\left.1^{-/-}\right)$or 1 (Stat $\left.1^{-/-}\right)$independent experiments.

IL-10eGFP ${ }^{+}$Tregs in tumor nodule-free lung tissue compared with normal lung may reflect extension of the inflammatory environment into this space. Overall, these results indicated that, independently of tumor type or location of growth, the TME contains a Treg population with an activated phenotype that is the major subset producing IL-10.

T cell IL-10 limits tumor Th17 inflammation. In order to evaluate the biological consequence of Treg activity in the TME, we examined the effects of IL-10 on inflammation through quantitation of IL-17-, IFN- $\gamma-$, and TNF-producing cell subsets in MC38 tumors derived from $\mathrm{Il10}^{-/-}$and WT mice along with T cell-conditional knockout $I l 10^{T \text { cell } \Delta / \Delta}$ mice and their respective $I l 10^{f l / f l}$ littermate controls (Figure 3). In vitro assay systems are poor evaluators of IL-10-dependent Treg activity (49). However, in vivo studies have demonstrated that Treg IL-10 production controls basal and inducible inflammation and Th17 cell activity at environmental interfaces such as the gut and lung $(42,50,51)$. Day 14 tumors in WT or $I l 10^{-/-}$mice were of similar size and had similar frequencies of infiltrating $\mathrm{CD} 45^{+}$leukocytes and similar frequencies of FoxP3 ${ }^{+}$ Tregs (Figure 3A and Supplemental Figure 3). However, Il10-/- and
Il10 $0^{T \text { cell } \Delta / \Delta}$ mice had significantly altered frequencies of polarized tumor-associated $\mathrm{CD}^{+}$and $\mathrm{CD}^{+} \mathrm{T}$ cells (identified using phorbol 12-myristate 13-acetate [PMA] and ionomycin stimulation and intracellular cytokine staining) compared with WT mice (Figure 3B). IL-17-producing Th17 and Tc17 cells were increased 4- to 5 -fold in tumors from $I l 10^{T \text { cell } \Delta / \Delta}$ mice compared with littermate controls (Th17, $P=0.004$; Tc17, $P=0.015$; Figure 3, B and C). Increases were also observed in complete $\mathrm{Il1O}^{-/-}$compared with WT mice, though this did not reach statistical significance for Tc17 cells (Figure 3B). A less than 2 -fold increase in total $\mathrm{CD}^{+}{ }^{+} \mathrm{T}$ cells and IFN- $\gamma$-producing Th1 cells was also observed in the absence of $\mathrm{T}$ cell IL-10. The frequencies of TNF-producing $\mathrm{CD}^{+} \mathrm{T}$ cells (but not $\mathrm{CD}^{+} \mathrm{T}$ cells) were also significantly increased in $\mathrm{Il10} \mathrm{T}^{\mathrm{T} \text { cell } \Delta / \Delta}$ mice compared with littermate $I l 10^{f l f l}$ controls $(P=0.012)$. Interestingly, $\mathrm{CD}^{+} \mathrm{T}$ cell frequencies were significantly lower in $\mathrm{Il10}^{-/-}$ mice compared with controls $(P=0.014)$, but not in $I l 10^{T \text { cell } \Delta / \Delta}$ mice, suggesting that the previously reported IL-10-dependent activation and tumor infiltration of $\mathrm{CD}^{+} \mathrm{T}$ cells (32) depends on non-T cell sources of IL-10. Confirming the relevance of $\mathrm{T}$ cell-produced IL-10 to T cell polarization, Il17 mRNA levels were 


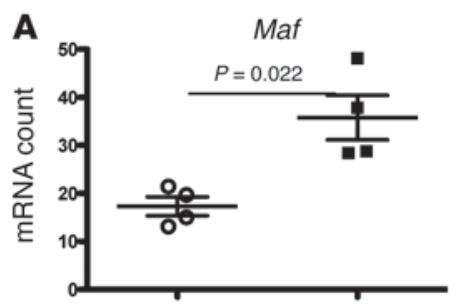

Icos
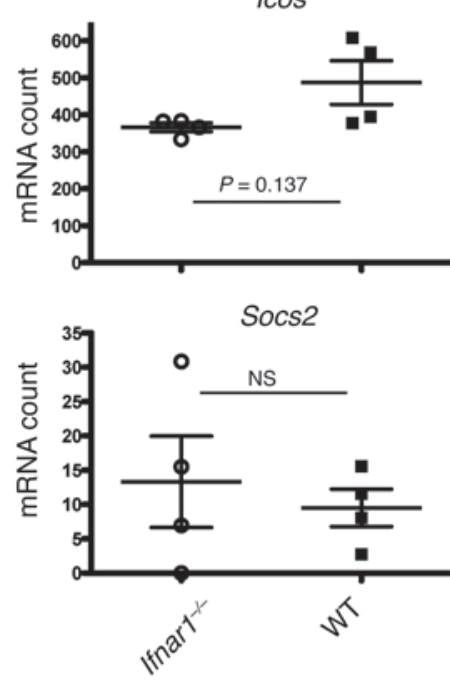

B

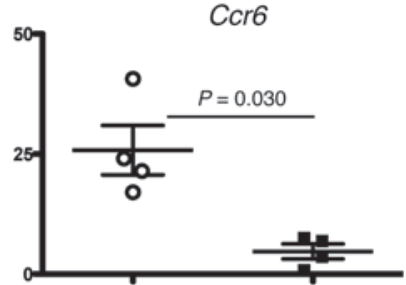

Ccr7
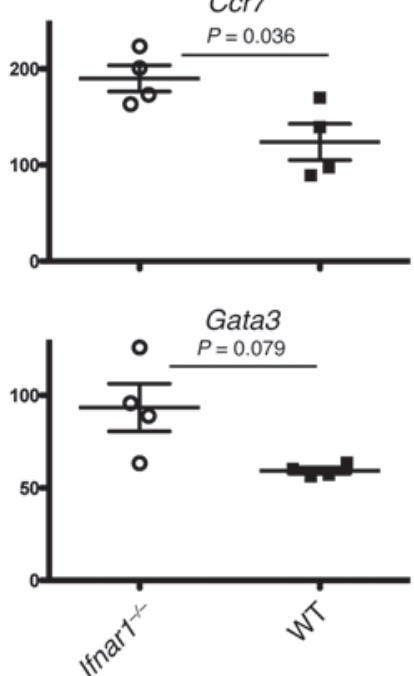
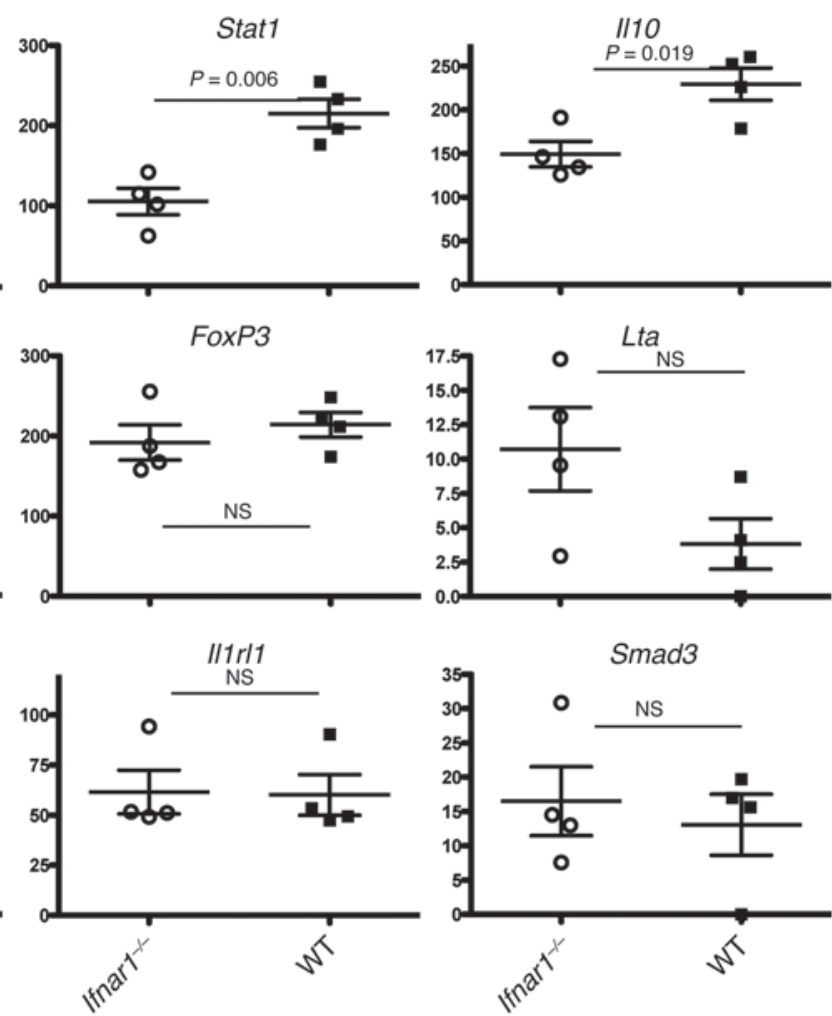

Sorted tumor Treg

C Bone Marrow

Ifnar1-l-

Stat1-1-

WT (or CD45.1)
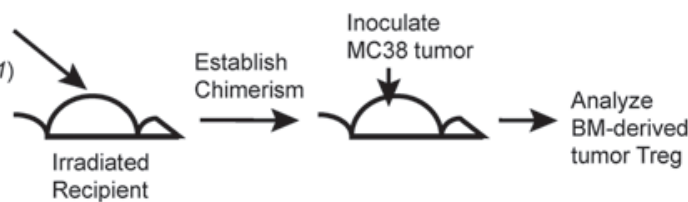

$\beta$-Actin

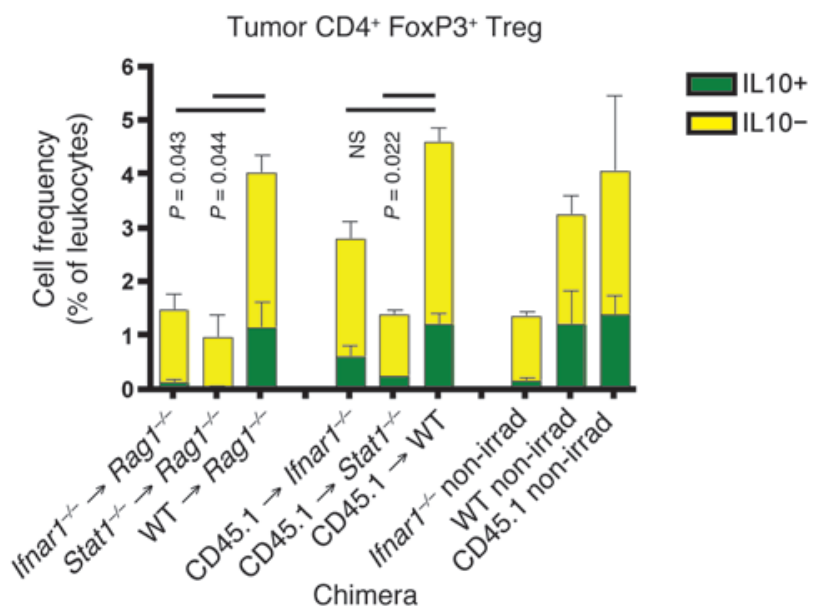

Figure 6

Type I IFN signaling is required for tumor Treg activation. (A) nCounter gene expression analysis of flow-sorted tumor Tregs from Ifnar1 $1^{-/-}$or WT mice. Data points show tumor Tregs from pools of mice flow sorted in independent experiments. Data show mean \pm SEM and $P$ value.

(B) Western analysis of STAT1 protein on whole spleen lysates of mice from indicated strains. Numbers above blot show STAT1 band intensities after normalization to $\beta$-actin. (C) Frequencies of Treg infiltrates in MC38 tumors from bone marrow chimeric mice and nonirradiated controls. Donor-to-host relationship (indicated with arrows) and cell frequencies are given with mean \pm SEM. $P$ values are given for indicated comparisons (by bar) of total Treg frequencies. 

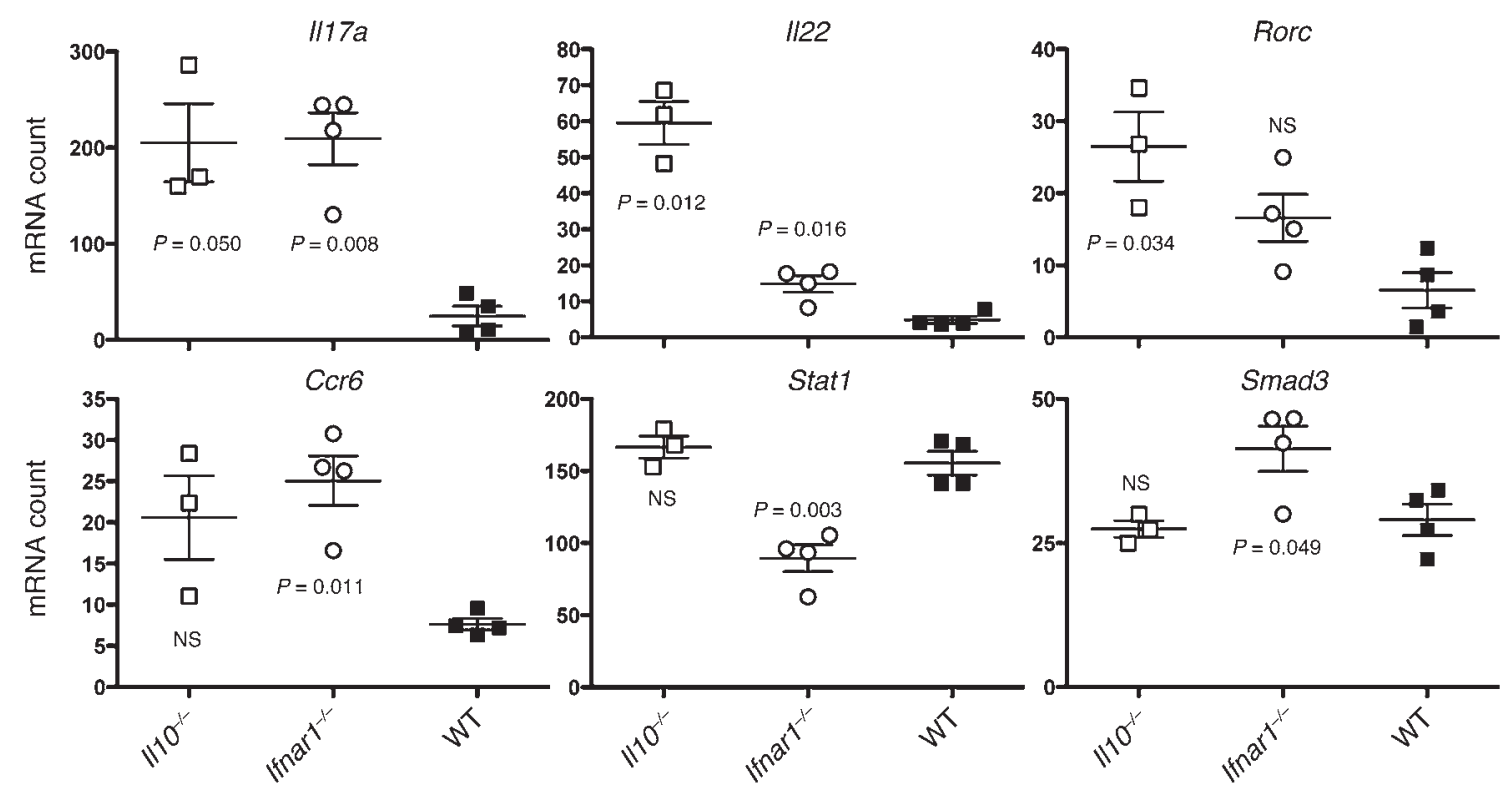

Figure 7

Ifnar1 and I/10 suppress Th17-associated gene expression by tumor CD4+ $T$ cells. CD4+CD25- T cells were sorted from MC38 tumor of indicated strain, and gene expression was analyzed by nCounter analysis. Each data point derives from an independent experiment using pools of mice. Mean \pm SEM and $P$-values are shown for Student's $t$ test with Welch's correction compared with WT cells.

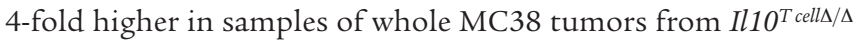
mice compared with littermate controls $(P<0.0001)$ and almost 14-fold higher in $I l 10^{-/-}$mice compared with WT mice (Figure 3D). The difference in Il17 mRNA expression between total $I l 10^{-/-}$mice and T cell-conditional $I l 10^{T \text { cell } \Delta / \Delta}$ mice was statistically significant, suggesting that IL-10 from non-T cells has an independent role in addition to Treg IL-10 (Figure 3D). A similar IL-10-dependent control of Il17 expression was also observed in B16-F10 melanoma (Supplemental Figure 2). To determine whether the presence of tumor was required for increased frequencies of Th17 cells in the absence of IL-10, we examined the frequencies of these cells in spleens of MC38 tumor-bearing and tumor-free mice (Supplemental Figure 4). Th17 cell frequencies were increased in spleen of tumor-bearing $(P=0.007)$ and, likewise, in tumor-free $(P<0.0001)$ $I l 10^{T \text { cell } \Delta / \Delta}$ mice compared with littermate $I l 10^{f / f l}$ controls by up to 4 -fold. This suggests that abrogation of T cell-derived IL-10 allows systemic expansion of Th17-polarized cells and that this expansion is independent of the presence of subcutaneous tumor. Therefore Treg IL-10 production limits IL-17 production by $\mathrm{CD}^{+}$ and $\mathrm{CD}^{+}$cells in the TME as well as systemically.

In order to test the relevance of IL-10 to Th17 regulation in human cancer, we analyzed publicly available gene expression microarray data sets. Despite poor sensitivity of microarray on solid tumor samples for cytokine gene expression, 2 head and neck squamous cell carcinoma (HNSCC) data sets were observed in which the low levels of IL10 and IL17 expression showed significant inverse correlation (Supplemental Figure 5), indicating that our observations in mouse are likely to relate to human cancer.

IL-10-expressing tumor Tregs have a highly activated phenotype. Multiple mechanisms have been identified that control IL-10 expression by different hematological cell populations, including regulation at the gene expression and at the posttranscriptional levels $(22,51-53)$. To investigate the mechanisms regulating
IL-10 expression in tumor Tregs, we compared the gene expression profile of spleen and tumor Tregs as well as of the IL-10+ ${ }^{+}$and IL- $10^{-}$populations of tumor Tregs. CD $4^{+}$Tregs were Flow-sorted from VERT-X mice using surface CD25 and TNFRII markers that we have shown to faithfully identify FoxP3 ${ }^{+}$cells in the TME of multiple mouse strains (Supplemental Figure 6), while the use of FoxP3-EGFP mice enabled comparison of these populations with canonical $\mathrm{CD}^{+}{ }^{+} \mathrm{FoxP}^{+}$Tregs. FACS-sorted populations of Tregs from spleen and tumor were analyzed by global gene expression microarrays (Figure 4A, Supplemental Figure 7, and Supplemental Table 1). Comparison of gene expression between IL-10eGFP ${ }^{+}$and IL-10eGFP- tumor Tregs from VERT-X mice indicated a limited number of differentially expressed genes (29 genes were overexpressed in $\mathrm{IL}^{-10 \mathrm{eGFP}}{ }^{+}$tumor Tregs and 88 underexpressed) (Figure 4A and Supplemental Table 1). Il10 was the most highly upregulated gene in the IL-10eGFP ${ }^{+}$subset $(25.04$-fold, $P=0.001$ ). Interestingly, many of the genes that were differentially regulated between IL-10eGFP ${ }^{+}$and IL-10eGFP ${ }^{-}$Tregs were similarly discordant between total tumor FoxP3EGFP ${ }^{+}$Tregs and total spleen FoxP3EGFP ${ }^{+}$Tregs (23/29 upregulated genes and 38/88 downregulated genes; Figure 4A). To obtain additional insight into gene expression associated with IL-10 function in Tregs, we analyzed gene expression patterns across the set of 3 spleen and 9 tumor microarrays for correlation to expression of Il10. Using a correlation requirement of $0.7,415$ genes positively, and 224 genes negatively correlated with $I$ l10. By filtering this set for those genes whose expression differed significantly between IL-10eGFP ${ }^{+}$and IL-10eGFP ${ }^{-}$tumor Tregs, we identified 48 genes that positively correlated and 24 genes that negatively correlated with Il10 (Supplemental Figure 8). Combining these 2 analyses, we identified a gene expression pattern in IL- $10^{+}$tumor Tregs that corresponds to an activated phenotype, including upregulation of Lag3, Prf1 (perforin), Gzmb (granzyme B), Entpd1 (CD39), Socs2, Cxcr6, and 

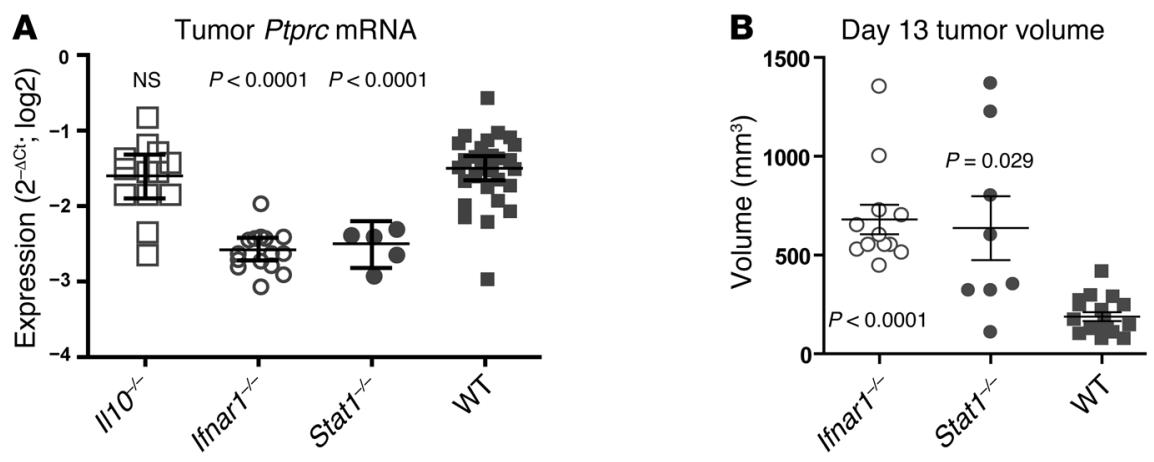

C
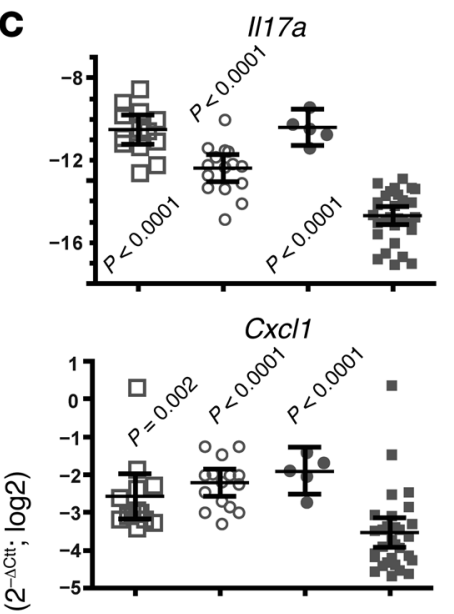

116
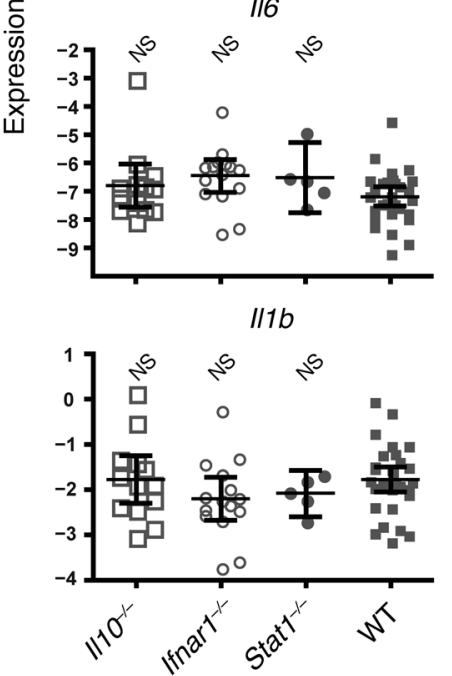

$1 / 22$
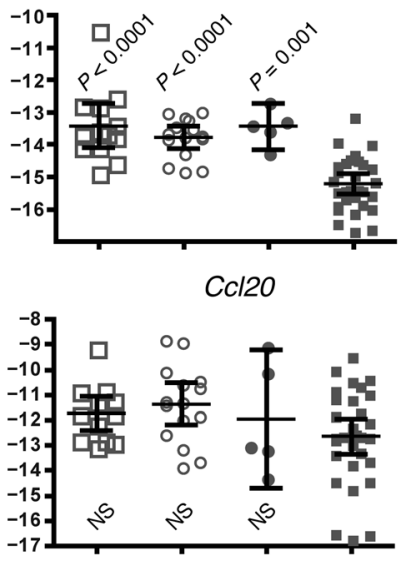

Prf1
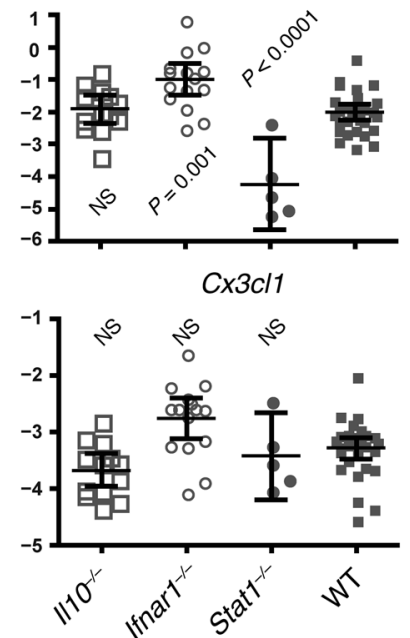

Rorc
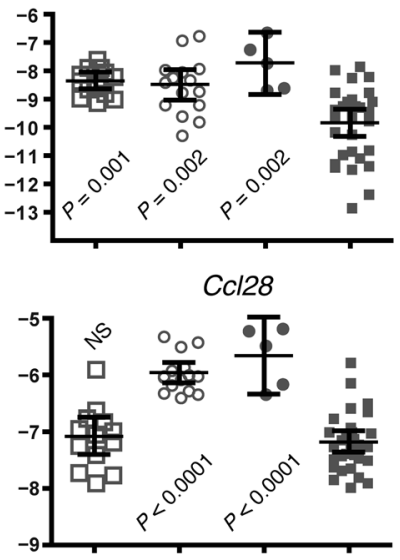

Gzmb
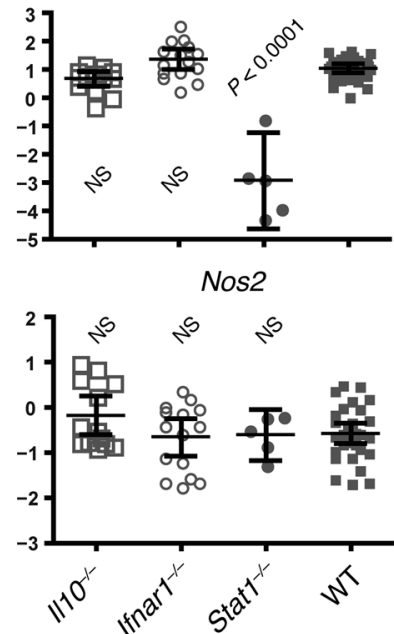

Figure 8

Th17-associated gene expression is limited by II10, Ifnar1, and Stat1 in the TME. (A) Real-time quantitative PCR for Ptprc (CD45) normalized to Hprt from whole-tumor tissue from indicated strains. Data from 4 to 6 independent experiments $\left(4, I / 10^{-1-} ; 4\right.$, Ifnar $1^{-1-} ; 1$, Stat $1^{-1-} ; 6$, WT) were combined and analyzed by mixed-effects ANOVA on log-transformed data. (B) Day 13 tumor volume. Statistics show mean \pm SEM and Student's $t$ test with Welch's correction. (C) Real-time quantitative PCR on whole-tumor cDNA for indicated genes normalized to Ptprc. Data from 4 to 6

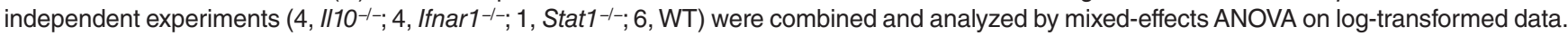
Geometric mean $\pm 95 \% \mathrm{Cl}$ and $P$ values for mixed-effects ANOVA (using Dunnett's method) are given for comparison with WT.

Maf (c-Maf) and downregulation of Ccr7 and Ccr6 (Figure 4A, Supplemental Table 1, and Supplemental Figure 8). Expression of Maf was highly correlated with that of $\operatorname{Il} 10(r=0.913$ and $r=0.815$ for independent probes; Supplemental Figure 8), and combined analysis of data from the 2 microarray probes for Maf showed that Maf was significantly overexpressed by IL- $10^{+}$tumor Tregs compared with the IL-10eGFP ${ }^{-}$subset (2-way ANOVA, $P=0.005$ ). As c-Maf is a known regulator of IL-10 that binds to the Il10 promoter and activates in multiple $\mathrm{T}$ cell types (54-57), it is also likely involved in driving Il10 expression in the IL- $10^{+}$tumor Tregs. 

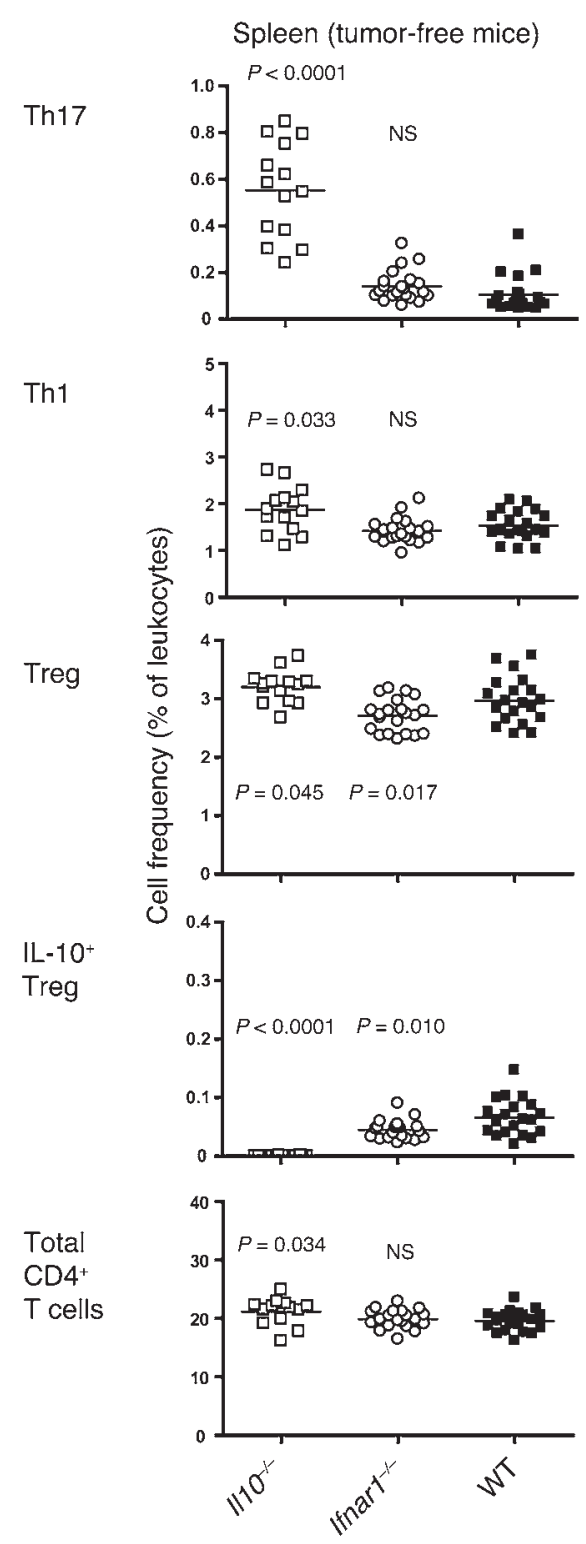

In order to validate the differences in gene expression detected by microarray, a predefined group of genes were analyzed using nCounter Analysis (Nanostring Technologies) on independently obtained tumor Treg samples (Figure 4B). These data confirmed an approximately 2 -fold greater Maf expression in IL-10eGFP ${ }^{+}$ tumor Tregs compared with IL-10eGFP ${ }^{-}$cells $(P=0.020)$ and overexpression of Icos. Conversely, downregulation of Ccr6, Ccr7, Lta, and $\mathrm{Smad} 3$ in IL-10eGFP ${ }^{+}$compared with IL-10eGFP- tumor Tregs was also observed (Figure 4B).

In addition to IL-10, Tregs utilize LAG3, perforin, granzyme B, and CD39 for their regulatory effector function (49), and their expression and activity has been associated with inflammatory conditions (5). Together, the minor gene expression differences, the restriction of IL-10 expression to the TNFRII ${ }^{+}$ subset, and the activated $\mathrm{T}$ cell signature on the $\mathrm{IL}-10^{+}$subset suggest that IL- $10^{+}$and IL- $10^{-}$tumor Tregs are 2 highly related populations of inflammatory Tregs that only differ by their degree of activation.

\section{Figure 9}

Frequencies of CD4+ $\mathrm{T}$ cells, Th1, Th17, and Tregs in spleens from tumor-free $/ 110^{-1-}$, Ifnar1 $1^{-1-}$, and WT mice. Single-cell suspensions from spleens of indicated strains of tumor-free mice were stimulated with PMA and ionomycin, and frequencies of IFN- $\gamma^{+} \mathrm{FoxP} 3-\mathrm{CD} 44^{+}$Th1 cells, IL17A+FoxP3-CD4+ Th17 cells, and IL-10+FoxP3+ Tregs were enumerated. Data are combined from 2 independent experiments. Frequencies are given as percentage of CD45+ leukocytes. Statistics show mean and $P$ values for 2-tailed unpaired Student's $t$ test with Welch's correction.

Stat1 and Ifnar1 are required for inflammatory induction of tumor Tregs. In order to determine the inflammatory pathways that contribute to IL-10 induction by Tregs in the TME, we compared frequencies of MC38 tumor-associated IL- $10^{+}$Tregs in mice lacking genes encoding selected proinflammatory molecules. IL-10 expression was detected in Tregs from these mouse strains by stimulation with PMA and ionomycin and intracellular staining, a procedure that detects frequencies of IL-10-positive Tregs similar to those observed in the VERT-X reporter strain (Figure 5A). Various IL-12 family members have been reported to drive IL-10 expression by lymphocytes, including IL-27 in Th1 cells (58), IL-12 in T and NK cells $(56,59,60)$, and IL-23 in Tregs (61). However, we observed no decrease in frequencies of IL- $10^{+} \mathrm{FoxP} 3^{+}$Tregs or

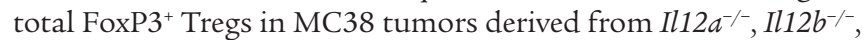
$\mathrm{Il} 23 \mathrm{a}^{-/-}$, or $\mathrm{Ws} \times \mathrm{1}^{-/-}$mice (Supplemental Figure 9), suggesting that IL-12, IL-23, IL-27, and IL-35 are not required for development of these cells. Total and IL- $10^{+}$tumor-associated Tregs were also observed at the same levels as found in WT mice in $\mathrm{Myd} 88^{-/-}$, Tnf ${ }^{--}$, $\mathrm{ICOS}^{-/-}$, and $\mathrm{IlO}^{-/-}$mice (Supplemental Figure 9). However, mice lacking certain STAT transcription factors had reduced frequencies of IL- $10^{+}$tumor Tregs. Stat $2^{-/-}$mice displayed a significant decrease $(P=0.007)$ in percentage of IL- $10^{+} \mathrm{FoxP}^{+}$Tregs within the $\mathrm{CD} 4^{+}$ $\mathrm{T}$ cell population (Supplemental Figure 9). T cell-specific Stat3 conditional knockout mice also showed a decrease in percentage of $\mathrm{IL}-10^{+} \mathrm{FoxP} 3^{+}$Tregs within the $\mathrm{CD} 4^{+} \mathrm{T}$ cell population $(P=0.022)$, but there was no difference in frequency of these cells when compared as a percentage of tumor $\mathrm{CD}^{4} 5^{+}$leukocytes (ns, $P=0.499$; Supplemental Figure 9). The most extreme phenotype was observed in Stat $1^{-1}$ mice that displayed very low frequencies of tumor Tregs in all but 1 experiment $(P<0.0001$; Figure 5B), with a trend toward an increase in total CD $4^{+} \mathrm{T}$ cell count $(P=0.100)$.

STAT1 activation is driven by JAK/STAT signaling downstream of multiple cytokine receptors including those for type I and type II IFN, IL-27, and IL-10. Ifnar1 $1^{-/-}$animals, which lack functional type I IFN signaling, but not Ifng $\mathrm{g}^{-/-}$animals (data not shown), showed reduced frequencies of tumor-associated IL-10+ ${ }^{+}$Tregs compared with WT animals $(P<0.0001$; Figure $5 \mathrm{~B})$. However, unlike in Stat $1^{-/-}$mice, the frequency of tumor-associated Tregs in Ifnar $1^{-/-}$ mice was inconsistently and nonsignificantly reduced, suggesting that STAT1 plays additional roles besides type I IFN signaling in regulation of tumor-associated Treg frequency. In order to confirm a role for type I IFN in induction of IL-10 by tumor Tregs while avoiding tumor dissociation and pharmacological stimulation, we compared the expression of $I l 10$ mRNA in whole tumors from Ifnar $1^{-/-}$and Stat $1^{-/-}$with that in WT mice and observed statistically significant 2.0- and 3.0-fold decreases, respectively, compared with WT (Figure 5C). Additionally, among sorted tumor Tregs, Ifnar1 $1^{-/}$tumor Tregs expressed lower levels of Maf and Il10, but higher levels of Cor6 and Cor 7 transcripts compared with WT Tregs (Figure 6A). The low frequency of Stat $1^{-/-}$tumor Tregs 
prevented us from performing a similar analysis on these cells. Therefore, Tregs in the Ifnar1 $1^{-/-}$TME mimicked the gene expression profile of the IL-10eGFP- ${ }^{-}$subset defined in VERT-X mice (Figure 4). It is noteworthy that, Ifnar $1^{-/-}$tumor Tregs expressed approximately half the level of Stat1 as WT Tregs (Figure 6A). Constitutive type I IFN signaling has previously been shown to maintain STAT1 expression $(62,63)$. We similarly observed a reduction in total STAT1 protein in Ifnar $1^{-/-}$spleen (Figure 6B). These observations suggest that type I IFN may in part control Treg number and activation by maintaining physiological levels of Stat 1 expression. Together, these data indicate that Ifnar 1 is required for tumor Tregs to undergo the inflammatory activation program and upregulation of Treg effector genes including Il10.

To determine whether activation of Tregs requires type I IFN signaling within Tregs in a cell-intrinsic manner, we analyzed tumor-associated Tregs obtained from bone marrow chimeric mice transplanted with bone marrow from Ifnar $1^{-/-}$or Stat $1^{-/-}$mice (Figure 6C). Ifnar $1^{-/-}$bone marrow was significantly less capable of generating tumor Tregs than WT bone marrow $(P=0.043)$, and few of these Tregs expressed IL-10. A similar reduction in Treg frequency was observed in chimeras bearing Stat $1^{-/-}$bone marrow. Interestingly, total $\mathrm{CD} 4^{+} \mathrm{T}$ cell frequencies were raised when Stat $1^{-/-}$bone marrow was transferred (data not shown), consistent with the observation in total Stat $1^{-/-}$mice (Figure 5B). Reciprocal transfers of WT bone marrow (CD45.1 congenic marker strain) into Ifnar $1^{-/}$, Stat $1^{-/}$, and WT hosts, showed nonsignificant reductions in Treg frequency when CD45.1 cells were transferred into Ifnar1 $1^{-/}$compared with WT hosts, but significantly lower total Tregs in the Stat $1^{-/-}$host compared with the WT host (Figure 6C). These data suggest that Ifnar1 and Stat1 in bone marrow-derived cells are required for generation of tumor-associated Tregs and for their production of IL-10. Stat1 expression in radio-resistant host cells is also important for Treg accumulation in the TME.

Ifnar 1 and Stat 1 limit Th17-type inflammation specifically in the TME. The observation that molecules involved in the type I IFN signaling pathway are required for accumulation and activation of Tregs in the TME (Figures 5 and 6) coupled with a requirement for Tregs and specifically Treg IL-10 to control Th17 responses (Figure 3 and refs. 41, 42, 64, 65), prompted us to address whether the type I IFN signaling pathway is required to control Th17 inflammation. Sorted $\mathrm{CD}^{+} \mathrm{CD} 25^{-} \mathrm{T}$ cells from tumors growing in Ifnar1 $1^{-/-}$mice showed elevated expression of Th17 genes including Il17a, Il22, and Cor6, compared with tumor-infiltrating $\mathrm{CD}^{+} \mathrm{T}$ cells in WT hosts (Figure 7). Elevated expression of these genes was also observed in tumor-infiltrating $\mathrm{CD}^{+} \mathrm{T}$ cells from $\mathrm{Il10}^{-/-}$mice. To confirm this increase in Th17-type inflammation among all tumor-infiltrating cells, we analyzed the expression of Th17-associated genes in whole

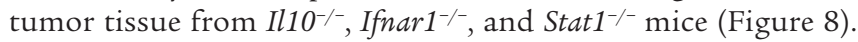
Infiltration of $\mathrm{CD} 45^{+}$leukocytes was significantly greater in tumors of Ifnar1 $1^{-/-}$and Stat $1^{-/-}$mice compared with WT as reflected by Ptprc (CD45) expression (Figure 8A), and this was associated with faster tumor growth in these strains (Figure 8B). Therefore, analysis of gene expression was performed using Ptprc as the normalizing control (Figure 8C). Significantly elevated expression of Il17a, Il22, and Rorc was observed in Ifnar ${ }^{-/}$and Stat $1^{-/-}$mice, similar to the observation in tumors growing in $\mathrm{Il}^{-/-}$mice (Figure 8C). Neutrophil-attracting Cxcl1 was significantly overexpressed in the Ifnar1 $1^{-/-}$, Stat $1^{-/-}$and Stat2 ${ }^{-/-}$TME compared with WT (Figure 8C and data not shown). In contrast, other Th17-associated genes, including Il6 and Gzmb, were not significantly elevated in Ifnar1-/- mice, and $I l 1 b$ and Nos 2 were unchanged (Figure 8C). The reduced expression of Prf1 and Gzmb observed in the TME of Stat $1^{-/-}$mice reflects a lack of infiltrating NK cells in this environment (data not shown). The increased expression of Th17-type genes and Th17 cells in Ifnar $1^{-/}$mice closely matched that in tumors from $\mathrm{Il}_{10^{-/-}}$ mice, suggesting a common mechanism of regulation in the TME. Similarly, Th17 inflammation is associated with increased angiogenesis that has been observed in tumors in both Ifnar $1^{-/-}$mice (66) and $I l 10^{T \text { cell } \Delta / \Delta}$ mice (Supplemental Figure 10).

The Ifnar1-dependent control of Th17 inflammation was specific to the TME, as a different pattern was observed in the spleens of tumor-free mice (Figure 9), with increased frequencies of Th17 and Th1 cells in $\mathrm{Il10}^{-/-}$mice, but not in Ifnar $\mathrm{I}^{-/-}$mice. Together, these data indicate that components of the type I IFN signaling pathway are required to limit Th17-type inflammation in the TME, but not in noninflamed tissues such as spleen, and that this control correlates with capacity of the type I IFN signaling pathway to drive accumulation and activation of Tregs.

\section{Discussion}

In this study, we examined the cell types and inflammatory signals responsible for production of IL-10 in the TME and the role IL-10 plays in the control of tumor inflammation. We observed that Tregs with an activated phenotype were the predominant IL-10-producing cells in the TME. Components of the type I IFN signaling pathway, including Ifnar1, Stat1, and Stat2, were required for accumulation and activation of Tregs and production of IL-10. Expression of these genes and Il10 itself was required for suppression of Th17type gene expression in tumors. Thus, type I IFN signaling forms an inflammatory network that controls Treg accumulation in tumors and their production of IL-10 and suppresses Th17-type inflammation in the TME (summarized in Supplemental Figure 11).

$\mathrm{CD}^{+} \mathrm{FoxP}^{+}$Tregs include natural thymus-derived Tregs that recognize self peptide-MHC or inducible Tregs that recognize foreign- or self peptide-MHC. Although inducible Tregs have been observed in experimental tumors that express potent exogenous antigen (67), a natural origin for the tumor-associated Tregs studied here is supported by several lines of evidence. First, the MC38 tumor line is B6 syngeneic and considered to be weakly immunogenic $(68,69)$, suggesting that self-encoded antigens are more relevant ligands for infiltrating Tregs. Second, though TGF- $\beta$ is required for inducible Treg conversion (70), antibody blockade of this cytokine during tumor growth reduced the growth rate but did not affect the abundance of Tregs or the fraction expressing IL-10 (data not shown). We observed that the phenotype of tumor-associated Tregs and particularly that of the IL-10-producing subset is consistent with inflammatory activation, as these cells express genes such as Maf and genes required for Treg effector functions, including those encoding IL-10, Granzyme B, Perforin, CD39, and Lag3 (ref. 5 and Figure 4). IL-10 expression by Tregs is also found in the gut, where chronic antigenic stimulation contributes to the activated Treg phenotype and can be experimentally mimicked by administration of the anti-CD3 antibody (46). Therefore, it is likely that chronic TCR stimulation of tumor-associated Tregs contributes to their activation and IL-10 production. As shown by this study, type I IFN signaling through Ifnar 1 and Stat $1 / 2$ is also required for tumor-associated Treg recruitment, activation, and IL-10 production. Combined, this evidence suggests that tumor-associated Tregs observed in our model originate from 
recruited natural Tregs that are selectively activated in TME by inflammatory conditions including self- or tumor-associated antigens and type I IFN signaling (71).

Type I IFN is well known for its antiviral effects and induction of antigen cross-presentation, but it also plays constitutive roles in specific pathogen-free conditions and can act in both an immunosuppressive and immunostimulatory manner (72-74). For example, IFN- $\beta$ treatment of multiple sclerosis patients induces expression of IL-10 that contributes to an immunosuppressive therapeutic effect $(75,76)$. IFN- $\beta$ is capable of inducing IL-10 expression in human and mouse FoxP3 ${ }^{-} \mathrm{T}$ cells in vitro and in vivo (data not shown and refs. 77, 78). Recently, it has been shown that type I IFN contributes to persistent viral infectious by inducing IL-10 production in $\mathrm{CD}^{+} \mathrm{T}$ cells and thus damping the antiviral immune response $(79,80)$. However, in many experimental conditions, IL-12 and IL-27, rather than type I IFN, have been described to be potent inducers of IL-10 production in effector T cells $(56,59,81)$. The precise regulatory mechanisms for IL-10 production by gutassociated Tregs remain unknown (22), whereas we show here for what we believe is the first time that high levels of IL-10 production also characterize tumor-associated Tregs and that type I IFN is responsible for this in the TME. In mouse transplantable tumors, type I IFN is induced through as as-yet-unidentified mechanism, and type I IFN signaling is required for antitumor $\mathrm{CD}^{+} \mathrm{T}$ cell responses (82). We have confirmed by real-time PCR that Ifnb transcripts are expressed in tumor tissue (data not shown). Type I IFN affects antitumor immune responses at different levels, in addition to the induction of IL-10 in tumor-associated Tregs. For example, type I IFN enhances antigen cross-presentation and production of immunomodulatory cytokines, particularly IL-12 $(83,84)$ and by acting directly on $\mathrm{CD}^{+} \mathrm{T}$ cells to enhance clonal expansion and memory formation (85). Although in this study we have focused on the role of Treg-dependent IL-10 production to control Th17 responses, IL-10 has also been shown to enhance the response of anti-tumor $\mathrm{CD}^{+} \mathrm{T}$ cells and their production of IFN- $\gamma$ (32) and, thus, it could also contribute to the described role of endogenous type I IFN to enhance antitumor $\mathrm{CD}^{+} \mathrm{T}$ cell responses (82).

Chimeric mice transplanted with Ifnar $1^{-/-}$or Stat $1^{-/-}$bone marrow displayed deficiency in abundance and IL-10 production of tumor Tregs (Figure 6), suggesting a cell-intrinsic requirement that was confirmed using Stat $1^{-/}$bone marrow in a mixed bone marrow chimera experiment (data not shown). However, the reduced frequency of tumor Tregs in Stat $1^{-/-}$mice transplanted with WT bone marrow indicates that STAT1 signaling is important in non-T cells and also in nonhematopoietic cells. Therefore, type I IFN signaling contributes to activation of Tregs within the TME through a mechanism that probably involves both direct type I IFN signaling on Tregs and indirect signaling through other cells. Type I IFN signaling may contribute to activation of tumor Tregs due to its ability to maintain STAT1 levels. Stat1 has been shown to be required for Treg-mediated control of Th17-mediated experimental autoimmune encephalitis (86) and we observed reduced Stat 1 transcript in tumor $\mathrm{CD}^{+} \mathrm{T}$ cells and Tregs and reduced STAT1 protein expression in spleens from Ifnar $1^{-/}$mice (Figure 6, A and B, and Figure 7), consistent with previous reports showing that constitutive IFN signaling maintains STAT1 and STAT2 expression $(62,63)$.

One possible explanation for differential accumulation and activation of tumor-associated Tregs might involve production of chemokines that attract Tregs to the tumor site. However, the Treg-attracting chemokines examined (Ccl20 and Ccl28; refs. $87,88)$ were overexpressed in tumors from Ifnar1 $1^{-/-}$and Stat1 $1^{-/-}$ mice (Figure 8). The activated IL-10-producing Tregs in the TME, however, had a characteristic expression of chemokine receptors, with high expression of CCR5 and CXCR6 and much decreased expression of CCR6 and CCR7 compared with non-IL-10-producing Tregs, and thus the mechanisms of recruitment of these Tregs in the tumor remain to be characterized.

Our data show that Treg IL-10 production controls the Th17 response in the TME, as has been previously shown in the intestine (89). The control of tumor IL-17 expression by IL-10 is also consistent with gene expression data from human HNSCC (Supplemental Figure 5). Our observation of enhanced Th17-type gene expression in MC38 tumor derived from IL-10-deficient and type I IFN signaling-deficient mice, combined with a requirement for Ifnar1, Stat1, and Stat2 for activation of tumor-associated Tregs and their production of IL-10, indicates that type I IFN controls the Th17 response at least in part by its effects on Tregs. However, the phenotype of Ifnar $1^{-/-}$and Stat $1^{-/-}$mice with regard to alterations in Th17 activity is different from that in IL-10-deficient strains, revealing mechanistic differences in control of Th17 function. In tumor-free spleens, total or T cell-specific IL-10 deficiency leads to increases in Th17 cells (Figure 9 and Supplemental Figure 4), indicating that T cell-derived IL-10 is important for control of the Th17 response not only in the TME but also systemically in steady state conditions. However, Ifnar $1^{-/-}$and WT spleens have similar frequencies of Th17 cells. This apparent discrepancy is explained by the observation that the frequencies of total and IL-10-producing Tregs in the spleen are unaffected by Ifnar 1 deficiency (Figure 9), and therefore maintain suppression of Th17 function in Ifnar $1^{-/}$mice. Thus, the role of type I IFN signaling in activating Tregs and their production of IL-10 is selective for the TME where Ifnar1/Stat1/Stat2 expression is required to suppress Th17-type inflammation through the induction of T cell-produced IL-10.

As expected, the functions of type I IFN and IL-10 do not completely overlap in the TME. Transplanted tumors grew faster in Ifnar1 $1^{-/}$, Stat $1^{-/}$, and Stat $2^{-/-}$mice than in WT animals, but in our experiments, the tumor growth rate in $\mathrm{Il10}^{-/-}$mice was not significantly greater than in WT (Figure 3, Figures 5-8, Supplemental Figure 3), though others have observed enhanced carcinogen-induced and transplantable tumor growth in $\mathrm{Il10}^{-/-}$mice (including the MC38 model) $(32,90)$. Type I IFN has many immunomodulatory effects in addition to the induction of IL-10. IFN inhibits angiogenesis by acting directly on tumor cells and on lymphocyte-induced vascular responses $(91,92)$. Interestingly, VEGF needs to downregulate type I IFN receptor expression in order to induce efficient angiogenesis (93). An additional explanation for greatly increased growth of several tumors including MC38 in Ifn $b^{-/-}$or Ifnar $1^{-/-}$mice has been ascribed to $\mathrm{Gr} 1^{+}$neutrophils that promote tumor angiogenesis and tumor growth in both Ifn $b^{-/-}$ and WT mice and are hyperactivated in the TME of $\mathrm{Ifn}^{-/-}$mice (66). Although these angiogenic mechanisms are not all activated in the absence of IL-10, the enhanced Th17 response can recruit neutrophils and is associated in our (Supplemental Figure 10) and other studies (36) with an increase in angiogenesis. Together, these data suggest that type I IFN may control Th17-type inflammation and angiogenesis through complementary mechanisms in the TME: suppression of neutrophils that are activated to promote angiogenesis in the absence of Ifnar1/Ifnb (66); and control of Treg activation and Treg production of IL-10, which limits Th17-type 
cytokine and chemokine expression as described by this study. Indeed, we observed that coadministration of Ifnar $1^{+/+}$Tregs, but not Ifnar $1^{-/-}$Tregs, with MC38 tumors substantially reduced tumor growth in Ifnar $1^{-/-}$hosts (data not shown). IL-10 and type I IFN signaling therefore play critical overlapping roles in limiting Th17 inflammation that may otherwise drive tumor growth or autoimmunity $(65,94)$. The precise relationship between these mechanisms remains to be clarified in future studies.

IL-10 is a potential therapeutic target for cancer therapy because blocking its function increases Th1 cytokine production under proinflammatory conditions. The evidence here shows that interference with IL-10 has the additional effect during normal tumor growth of inhibiting Th17 inflammation that may otherwise promote tumor growth. Combined with the recent description of IL-10-promoting antitumor $\mathrm{CD}^{+} \mathrm{T}$ cell responses (32) and earlier evidence that administration or overexpression of IL-10 can drive antitumor functions (31), this new model indicates that therapeutic blockade of IL-10 signaling is likely to promote tumor inflammation and tumor growth and should only be applied during a short window when proinflammatory, Th1-promoting responses can be induced. Similarly, Tregs may be poor therapeutic targets in tumors where they suppress Th17-type inflammation. Conversely, type I IFN can slow tumor growth in patients (see refs. 66, 72) and is required for cross-presentation of tumor antigens $(82,95)$, but its capacity to promote Treg activation and expression of IL-10 described herein may counteract Th1 antitumor responses. A clear understanding of this complex network of immunosuppressive regulators will be critical to generating highly effective immunotherapeutic protocols against cancer.

\section{Methods}

Mice. All animals were maintained in a specific pathogen-free environment (also free of helicobacter and parvovirus) on a B6 background and were sexand age-matched for experiments, typically between 8 and 14 weeks of age. WT C57BL/6Ncr and B6.CD45.1 congenic mice were obtained from the Frederick National Laboratory Animal Production Program. All knockout strains were obtained with or crossed onto a B6 background. Information on genetically modified mouse strains is given in Supplemental Methods. CD4-Cre mice were obtained from Taconic Farms and were crossed with $I l 10^{f / / f l}, I l 10 r^{l / f l}$, and Stat $3^{f / / f l}$ to generate $I l 10^{T \text { cell } \Delta / \Delta}, I l 10 r^{T \text { cell } L / \Delta}$, and Stat $3^{T \text { cell } \Delta / \Delta}$ strains. Homozygous flox (fl/fl) and, in a few cases, hemizygous flox (fl/+) genotype littermates that lacked the Cre gene were used as routine controls for conditional knockout mice in order to minimize variation in the microbiota and genetic background. C57BL/6J, Il6 $6^{-/}, I l 12 \mathrm{a}^{-/}, \mathrm{Il} 12 \mathrm{~b}^{-/-}$, $\mathrm{Icos}^{-/}, \mathrm{Rag}^{1^{-/}}$, and $\mathrm{Tnf} \mathrm{f}^{--}$mice were obtained from the Jackson Laboratory. Mice were crossed and housed by the Laboratory Animal Sciences Program, Frederick National Laboratory.

Tumor studies and organ harvest. MC38 colon carcinoma line (68, 96), B16-F10 melanoma (97), and Lewis lung carcinoma (98) were obtained from Robert Wiltrout and the Developmental Therapeutics Program Tumor Repository (Frederick National Laboratory). Cells were maintained in vitro and inoculated subcutaneously in the right flank at 1 to $5 \times 10^{5}$ cells or intravenously at $2.5 \times 10^{5}$ cells for B16-F10 study. Tumors were measured by caliper and volume calculated as $\left(0.5 \times\right.$ length $\times$ width $\left.^{2}\right)$. Mice were harvested at day $12-16$ (subcutaneous) or day 20 (intravenous), organs dissected, and single-cell suspensions prepared from tumor tissue by digestion of approximately 1 - to $2-\mathrm{mm}$ pieces with $200 \mathrm{U} / \mathrm{ml}$ Collagenase IV (Invitrogen) and $100 \mu \mathrm{g} / \mathrm{ml}$ DNase I (Roche) in RPMI medium with 0.5\% FBS for 2 hours with pipetting (tituration) every half hour. Digestion mixes were passed through a filter and mechanically disrupted to obtain a single-cell suspen- sion and DNase I/collagenase neutralized by addition of 2 mM EDTA. Following washing in RPMI with $10 \%$ FBS, cells were directly used for flow cytometric staining or cell culture. For spleen and lymph node, single-cell suspensions were obtained by mechanical disruption and ACK lysis (for spleen). For analysis of lung, a dissection microscope was used to separate B16-F10 tumor nodules from nonnodule tissue where applicable, and lobes of lung were digested in collagenase/DNase I for 1 hour. Small intestine and large intestine intraepithelial leukocyte and lamina propria cells were obtained as previously described (99).

Bone marrow chimeras. Bone marrow chimeras were established in indicated recipient strains by intravenous inoculation of $10^{7}$ bone marrow cells from donor strains in $950 \mathrm{cGy} \gamma$-irradiated recipients. Chimeric mice were given acid water containing $0.5 \mathrm{mg} / \mathrm{l}$ amoxicillin for 1 week following bone marrow transfer and returned to normal housing for at least 2 months prior to tumor inoculation.

Flow cytometric analysis. For surface staining, single-cell suspensions were resuspended in FACS buffer (PBS, 2 mM EDTA, 1\% FBS) and blocked with $10 \mu \mathrm{g} / \mathrm{ml} 2.4 \mathrm{G} 2,2 \%$ normal mouse serum prior to staining in blocking buffer. LIVE/DEAD-Aqua vital dye (Invitrogen) was used to exclude dead cells in most experiments. Anti-CD45 was included in tumor-cell stain to identify leukocytes. Intracellular staining for $\mathrm{FoxP}^{+}$Tregs on fresh cells was performed using a FoxP3 Staining Buffer Set (eBioscience). Identification of cytokine-competent Tregs (FoxP3+IL-10+), Th1 (CD4+IFN- $\left.\gamma^{+}\right)$, Th17 $\left(\mathrm{CD} 4+\mathrm{IL}-17 \mathrm{~A}^{+}\right), \mathrm{CD} 4{ }^{+} \mathrm{TNF}^{+}$, and $\mathrm{CD} 8^{+} \mathrm{Tc} 1$ and $\mathrm{Tc} 17$ cells was performed following ex vivo stimulation with PMA and ionomycin. Single-cell suspensions of tumor cells were resuspended in IMDM containing 5 pg/ml PMA (Sigma-Aldrich), $5 \mathrm{ng} / \mathrm{ml}$ ionomycin (Sigma-Aldrich), and 1/1000 brefeldin A (Golgiplug; BD) for 4 to 5 hours at $37^{\circ} \mathrm{C}$. For spleen cells, $0.75 \mathrm{ng} / \mathrm{ml}$ ionomycin was used. Following culture, cells were stained with LIVE/ DEAD-Aqua dye (Invitrogen) and fixed with $2 \%$ paraformaldehyde in PBS; this was followed by blocking and intracellular staining in FACS buffer containing $0.5 \%$ saponin (Sigma-Aldrich). Antibodies used for flow cytometry are detailed in Supplemental Methods. Cells were acquired using an LSR II (BD) and data analyzed using FlowJo (Tree Star).

FACS-based cell sorting. For FACS sorting, single-cell suspensions from an MC38 tumor were obtained as described for Tumor studies above with the modifications that mice were perfused with PBS prior to tumor harvest. Single-cell suspensions were further purified by centrifugation at $800 \mathrm{~g}$ on $40 \% / 80 \%$ Percoll (adjusted to 0.29 osmoles/1; GE Healthcare) for 20 minutes or the Dead Cell Removal Kit (Miltenyi Biotech) according to the manufacturers' instructions. Cells were stained and $\mathrm{CD}^{+} \mathrm{T}$ cells identified by gating on $\mathrm{FSC} / \mathrm{SSC} / \mathrm{CD} 45^{+} \mathrm{CD} 11 \mathrm{~b}^{\text {lo }} \mathrm{CD}^{+} \mathrm{CD}^{+}$cells. Treg populations from FoxP3-EGFP mice were obtained by further gating FoxP3EGFP ${ }^{+}$ cells, while Tregs from VERT-X mice were gated on CD $25^{+} \mathrm{CD} 120 \mathrm{~b}^{+/ 10}$ cells and subgated for IL-10eGFP ${ }^{+}$and IL-10eGFP- subsets. Sorting was performed on an Aria II Flow Sorter (BD) directly into RLT lysis buffer (QIAGEN) for gene expression analysis.

Microarray analysis. Microarray analysis was performed by Genus Biosystems. RNA was purified by RNeasy (QIAGEN) and quality control tested by Bioanalyzer (Agilent). First- and second-strand cDNA were prepared from RNA, followed by cRNA preparation and fragmentation. cRNA was hybridized to Agilent Whole Genome $4 \times 44 \mathrm{~K}$ Arrays, and slides were washed and scanned on an Agilent G2565 Microarray Scanner followed by data analysis using Agilent Feature Extraction.

Three samples of VERTX IL-10eGFP ${ }^{+}$tumor tregs were compared with IL-10eGFP- Tregs. All nonexpressed genes were removed by eliminating probes for which fewer than 2 samples gave signals in both cell subsets. NIA Array Tools software (100) was used to determine differentially expressed genes. Gene expression was normalized (quartile) and $\log _{10}$ transformed. ANOVA, using intensity-specific average (average model) error variance 
was used to obtain $P$ values and set a threshold at $P<0.01$. To remove highly variable probes, significant probes were refiltered for estimated variance based on each probe (Actual Model) for probes with $P<0.1$. Correlation analysis was performed using microarray data from triplicate arrays of all 4 Treg populations (VERT-X tumor IL-10eGFP ${ }^{+}$, VERT-X tumor IL-10eGFP $^{-}$, tumor FoxP3EGFP', spleen FoxP3EGFP ${ }^{+}$) or from tumorderived arrays alone. Expression patterns of each probe were correlated to that of IL-10 using NIA Array Tools using a correlation coefficient cut-off of 0.7 and fold-difference threshold of 1.5. Microarray data are available from the GEO (GSE49380).

Gene expression quantitation by real-time PCR. Tissues were preserved in RNAlater (QIAGEN) and kept at $-80^{\circ} \mathrm{C}$ following harvest. RNeasy (QIAGEN) was used to extract RNA from whole-tumor samples, following mechanical disruption in RLT buffer (QIAGEN) using 1-mm diameter glass beads and a Mini-Beadbeater (Biospec Products). RNA extraction by RNeasy (QIAGEN) was performed according to the manufacturer's instructions with on-column DNase I digestion. cDNA was prepared using the High Capacity cDNA Reverse Transcription Kit (Life Technologies) using $1 \mu \mathrm{g}$ of total RNA, random primers, and RNAse Inhibitor according to the manufacturer's instructions. Real-time PCR was performed on a StepOnePlus Real-Time PCR device (Life Technologies) with TaqMan Gene Expression Master Mix (Life Technologies), 50 ng RNA equivalent of cDNA, and TaqMan probe/primer assays as detailed in Supplemental Methods. Forty cycles of 15 seconds at $95^{\circ} \mathrm{C}$ (melting) followed by 60 seconds at $60^{\circ} \mathrm{C}$ (annealing/extension) were performed. Gene expression was calculated

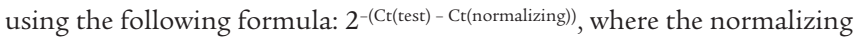
gene was Hprt for most experiments and Ptprc for experiments shown in Figure 5C, Figure 8C, and Supplemental Figure 2. When gene expression was not detected, Ct was set at the maximum cycle number of 40 to facilitate statistical comparison.

Gene expression quantitation by $n$ Counter analysis. nCounter analysis (NanoString Technologies) was used to analyze gene expression in FACSsorted $\mathrm{CD} 4{ }^{+} \mathrm{CD} 25^{-} \mathrm{T}$ cells and $\mathrm{CD} 4^{+} \mathrm{CD} 25^{+} \mathrm{CD} 120 \mathrm{~b}^{+} /$low Tregs. Between 5,000 and 50,000 cells were FACS sorted into RLT buffer, and RNA was extracted using the RNEasy Micro Kit (QIAGEN) according to the manufacturer's instructions. RNA was hybridized with customized Reporter CodeSet and Capture ProbeSet (NanoString Technologies) designed against sequences listed in Supplemental Table 2. The mRNA molecules were counted with the NanoString nCounter at the Laboratory of Molecular Technology (Advance Technology Program, Frederick National Laboratory). To normalize data, average background was subtracted from raw count, and normalization was performed using the geometric mean count from 4 housekeeping genes (Gapdh, Hprt, Rpl9, and Rpl30).

Western immunoblot analysis. Spleen lysates were prepared in RIPA buffer, and $40 \mu \mathrm{g}$ was loaded and separated by Tris-Glycine SDS-PAGE. Following transfer to nitrocellulose membrane and blocking with $5 \%$ BSA, targets were probed with anti-STAT1 (Cell Signaling Technology) and anti-mouse IgGHRP (GE Healthcare) or anti-actin-HRP (Calbiochem). ECL Prime Western Blotting Detection reagents (GE Healthcare) and the ChemiDoc XRS and Quantity One software (Bio-Rad) were used to detect and acquire images.
Statistics. Data were plotted using Prism (GraphPad), and routine analysis performed using unpaired 2-tailed Student's $t$ test with Welch's correction for unequal variances. Box-and-whiskers plots show median, 25th, and 75th percentiles (bounds of box), with Tukey whiskers showing the minimum (or 25 th percentile minus $1.5 \times$ interquartile range, whichever is the greatest), maximum (or 75 th percentile plus $1.5 \times$ interquartile range, whichever the least), and outliers, if present. Data shown in Figure 1E, Figure 3D, Figure 5, $\mathrm{B}$ and $\mathrm{C}$, and Figure $8 \mathrm{~A}$ were combined from multiple experiments and analyzed in SAS (SAS version 9.1, SAS Institute) by mixed-effects ANOVAs that assumed experiment and litter were random effects and that age was a covariate. Data were transformed prior to analysis (log transformation, Figure $1 \mathrm{E}$ and Figure 3D; arcsine transformation, Figure $5 \mathrm{~B}$; $\log _{2}$ transformation, Figure $5 \mathrm{C}$ and Figure $8 \mathrm{~A}$; and $\log _{2}$ transformation or a reciprocal $-1 / \mathrm{x}$ transformation, Figure $8 \mathrm{C}$ ) and $P$ values calculated using multiple comparisons procedures by Tukey-Kramer (Figure 1E and Figure 3D) and by Dunnett (Figure 5C, Figure 8, A and C). $P<0.05$ was considered significant.

Study approval. Animal studies were approved by the IACUC of the National Cancer Institute and were conducted in accordance with the IACUC guidelines and the NIH Guide for the Care and Use of Laboratory Animals (NIH publication no. 86-23. Revised 1985).

\section{Acknowledgments}

We wish to thank Dragana Jankovic, Yasmine Belkaid, Amiran Dzutsev, Romina Goldszmid, Marco Cardone, Elena Riboldi, Rosalba Salcedo, Xin Chen, Cristina Bergamaschi, Howard Young, and Andy Hurwitz for their insight and discussion. We thank Robin Winkler-Pickett and Tim Back for expert technical assistance. We are very grateful to Kathleen Noer and Roberta Matthai of the Cancer and Inflammation Program Flow Cytometry Core for assistance with flow sorting. We also thank Bob Jack, Brian Kelsall, Christian Schindler, Vijay Kuchroo, David Levy, Michel Aguet, Sergei Nedospasov, Shizuo Akira, Christian Saris, and Amgen Inc. for their kind provision of mice. This work was supported by the Intramural Research Program of the National Cancer Institute, NIH (NCI/NIH) under contract no. HHSN261200800001E. The content of this publication does not necessarily reflect the views or policies of the Department of Health and Human Services, nor does mention of trade names, commercial products, or organizations imply endorsement by the US government.

Received for publication June 4, 2012, and accepted in revised form August 6, 2013.

Address correspondence to: Giorgio Trinchieri, Director, Cancer and Inflammation Program, Center for Cancer Research, NCI, NIH, Bldg. 560/Room 31-93, Frederick, Maryland 21702-1201, USA. Phone: 301.846.1323; Fax: 301.846.1673; E-mail: trinchig@mail.nih.gov.

Christopher L. Karp's present address is: The Bill and Melinda Gates Foundation, Seattle, Washington, USA.
1. Grivennikov SI, Greten FR, Karin M. Immunity, inflammation, and cancer. Cell. 2010;140(6):883-899.

2. Hanahan D, Weinberg RA. Hallmarks of cancer: the next generation. Cell. 2011;144(5):646-674

3. Hurwitz AA, Watkins SK. Immune suppression in the tumor microenvironment: a role for dendritic cell-mediated tolerization of T cells. Cancer Immunol Immunother. 2012;61(2):289-293.

4. Josefowicz SZ, Lu LF, Rudensky AY. Regulatory T cells: mechanisms of differentiation and function. Annu Rev Immunol. 2012;30:531-564.

5. Yamaguchi T, Wing JB, Sakaguchi S. Two modes of immune suppression by Foxp3(+) regulatory $\mathrm{T}$ cells under inflammatory or non-inflammatory conditions. Semin Immunol. 2011;23(6):424-430.

6 . Bennett CL, et al. The immune dysregulation, polyendocrinopathy, enteropathy, X-linked syndrome (IPEX) is caused by mutations of FOXP3. Nat Genet. 2001;27(1):20-21.

7. Wildin RS, et al. X-linked neonatal diabetes mellitus, enteropathy and endocrinopathy syndrome is the human equivalent of mouse scurfy. Nat Genet. 2001;27(1):18-20.

8. Chaudhry A, et al. CD4+ regulatory T cells control
TH17 responses in a Stat3-dependent manner. Science. 2009;326(5955):986-991.

9. Josefowicz SZ, et al. Extrathymically generated regulatory $\mathrm{T}$ cells control mucosal TH2 inflammation. Nature. 2012;482(7385):395-399.

10. Koch MA, Tucker-Heard G, Perdue NR, Killebrew JR, Urdahl KB, Campbell DJ. The transcription factor T-bet controls regulatory $\mathrm{T}$ cell homeostasis and function during type 1 inflammation. Nat Immunol. 2009;10(6):595-602.

11. Wohlfert EA, et al. GATA3 controls Foxp3(+) regulatory $\mathrm{T}$ cell fate during inflammation in mice. 
J Clin Invest. 2011;121(11):4503-4515.

12. Klages K, et al. Selective depletion of Foxp3+ regulatory $\mathrm{T}$ cells improves effective therapeutic vaccination against established melanoma. Cancer Res. 2010;70(20):7788-7799.

13. Stagg J, et al. CD73-deficient mice have increased antitumor immunity and are resistant to experimental metastasis. Cancer Res. 2011;71(8):2892-2900.

14. Teng MW, Ngiow SF, von Scheidt B, McLaughlin $\mathrm{N}$, Sparwasser T, Smyth MJ. Conditional regulatory T-cell depletion releases adaptive immunity preventing carcinogenesis and suppressing established tumor growth. Cancer Res. 2010;70(20):7800-7809.

15. Teng MW, Ritchie DS, Neeson P, Smyth MJ. Biology and clinical observations of regulatory $T$ cells in cancer immunology. Curr Top Microbiol Immunol. 2011; 344:61-95.

16. Teng MW, et al. Multiple antitumor mechanisms downstream of prophylactic regulatory T-cell depletion. Cancer Res. 2010;70(7):2665-2674

17. Menetrier-Caux C, Curiel T, Faget J, Manuel M, Caux C, Zou W. Targeting regulatory T cells. Target Oncol. 2012;7(1):15-28.

18. D'Andrea A, Aste-Amezaga M, Valiante NM, Ma X, Kubin M, Trinchieri G. Interleukin 10 (IL-10) inhibits human lymphocyte interferon gammaproduction by suppressing natural killer cell stimulatory factor/IL-12 synthesis in accessory cells. J Exp Med. 1993;178(3):1041-1048.

19. Fiorentino DF, et al. IL-10 acts on the antigen-presenting cell to inhibit cytokine production by Th 1 cells. J Immunol. 1991;146(10):3444-3451.

20. Moore KW, de Waal Malefyt R, Coffman RL, O'Garra A. Interleukin-10 and the interleukin-10 receptor. Annu Rev Immunol. 2001;19:683-765.

21. Sanjabi S, Zenewicz LA, Kamanaka M, Flavell RA. Anti-inflammatory and pro-inflammatory roles of TGF-beta, IL-10, and IL-22 in immunity and autoimmunity. Curr Opin Pharmacol. 2009;9(4):447-453.

22. Saraiva M, O'Garra A. The regulation of IL-10 production by immune cells. Nat Rev Immunol. 2010; 10(3):170-181.

23. Guiducci C, Vicari AP, Sangaletti S, Trinchieri G, Colombo MP. Redirecting in vivo elicited tumor infiltrating macrophages and dendritic cells towards tumor rejection. Cancer Res. 2005; 65(8):3437-3446

24. Kortylewski M, et al. Inhibiting Stat3 signaling in the hematopoietic system elicits multicomponent antitumor immunity. Nat Med. 2005;11(12):1314-1321.

25. Lu H, et al. Treatment failure of a TLR-7 agonist occurs due to self-regulation of acute inflammation and can be overcome by IL-10 blockade. JImmunol. 2010;184(9):5360-5367.

26. Vicari AP, Caux C, Trinchieri G. Tumour escape from immune surveillance through dendritic cell inactivation. Semin Cancer Biol. 2002;12(1):33-42.

27. Wang $T$, et al. Regulation of the innate and adaptive immune responses by Stat- 3 signaling in tumor cells. Nat Med. 2004;10(1):48-54.

28. Calzada-Wack JC, Frankenberger M, ZieglerHeitbrock HW. Interleukin-10 drives human monocytes to CD16 positive macrophages. J Inflamm. 1996;46(2):78-85.

29. Rahimi AA, Gee K, Mishra S, Lim W, Kumar A. STAT-1 mediates the stimulatory effect of IL-10 on CD14 expression in human monocytic cells. JImmunol. 2005;174(12):7823-7832.

30. Berg DJ, et al. Enterocolitis and colon cancer in interleukin-10-deficient mice are associated with aberrant cytokine production and CD4(+) TH1like responses. J Clin Invest. 1996;98(4):1010-1020.

31. Mocellin S, Marincola FM, Young HA. Interleukin-10 and the immune response against cancer: a counterpoint. J Lenkoc Biol. 2005;78(5):1043-1051.

32. Mumm JB, et al. IL-10 elicits IFNgamma-dependent tumor immune surveillance. Cancer Cell. 2011; 20(6):781-796.
33. Sellon RK, et al. Resident enteric bacteria are necessary for development of spontaneous colitis and immune system activation in interleukin-10-deficient mice. Infect Immun. 1998;66(11):5224-5231.

34. Martin F, Apetoh L, Ghiringhelli F. Controversies on the role of Th17 in cancer: a TGF-beta-dependent immunosuppressive activity? Trends Mol Med. 2012; 18(12):742-749.

35. Middleton GW, Annels NE, Pandha HS. Are we ready to start studies of Th17 cell manipulation as a therapy for cancer? Cancer Immunol Immunother. 2012; 61(1):1-7.

36. Numasaki $M$, et al. Interleukin-17 promotes angiogenesis and tumor growth. Blood. 2003 101(7):2620-2627.

37. Ouyang W, Kolls JK, Zheng Y. The biological functions of $\mathrm{T}$ helper 17 cell effector cytokines in inflammation. Immunity. 2008;28(4):454-467.

38. Gregory AD, Houghton AM. Tumor-associated neutrophils: new targets for cancer therapy. Cancer Res. 2011;71(7):2411-2416.

39. Kryczek I, et al. Phenotype, distribution, generation, and functional and clinical relevance of Th17 cells in the human tumor environments. Blood. 2009; 114(6):1141-1149.

40. Muranski P, et al. Tumor-specific Th17-polarized cells eradicate large established melanoma. Blood. 2008;112(2):362-373.

41. Chaudhry A, et al. Interleukin-10 signaling in regulatory $\mathrm{T}$ cells is required for suppression of Th17 cell-mediated inflammation. Immunity. 2011; 34(4):566-578

42. Huber S, et al. Th17 cells express interleukin-10 receptor and are controlled by Foxp3 and Foxp3+ regulatory CD4+ T cells in an interleukin-10-dependent manner. Immunity. 2011;34(4):554-565.

43. Madan R, et al. Nonredundant roles for B cellderived IL-10 in immune counter-regulation. J Immunol. 2009;183(4):2312-2320.

44. Chen X, Subleski JJ, Kopf H, Howard OM, Mannel DN, Oppenheim JJ. Cutting edge: expression of TNFR2 defines a maximally suppressive subset of mouse CD4+CD25+FoxP3+ T regulatory cells: applicability to tumor-infiltrating $\mathrm{T}$ regulatory cells. J Immunol. 2008;180(10):6467-6471.

45. Atarashi $\mathrm{K}$, et al. Induction of colonic regulatory $\mathrm{T}$ cells by indigenous Clostridium species. Science. 2011;331(6015):337-341.

46. Kamanaka M, et al. Expression of interleukin-10 in intestinal lymphocytes detected by an interleukin-10 reporter knockin tiger mouse. Immunity. 2006;25(6):941-952.

47. Kitamura K, Farber JM, Kelsall BL. CCR6 marks regulatory $\mathrm{T}$ cells as a colon-tropic, IL-10-producing phenotype. J Immunol. 2010;185(6):3295-3304.

48. Maynard CL, et al. Regulatory $\mathrm{T}$ cells expressing interleukin 10 develop from Foxp3+ and Foxp3precursor cells in the absence of interleukin 10. Nat Immunol. 2007;8(9):931-941.

49. Vignali DAA, Collison LW, Workman CJ. How regulatory T cells work. Nat Rev Immunol. 2008; 8(7):523-532.

50. Kullberg MC, et al. Bacteria-triggered CD4(+) T regulatory cells suppress Helicobacter hepaticusinduced colitis. J Exp Med. 2002;196(4):505-515.

51. Liu Y, et al. MicroRNA-98 negatively regulates IL-10 production and endotoxin tolerance in macrophages after LPS stimulation. FEBS Lett. 2011; 585(12):1963-1968.

52. Mosser DM, Zhang X. Interleukin-10: new perspectives on an old cytokine. Immunol Rev. 2008; 226:205-218

53. Sharma A, et al. Posttranscriptional regulation of interleukin-10 expression by hsa-miR-106a. Proc Natl Acad Sci U S A. 2009;106(14):5761-5766.

54. Apetoh L, et al. The aryl hydrocarbon receptor interacts with c-Maf to promote the differentiation of type 1 regulatory T cells induced by IL-27. Nat
Immunol. 2010;11(9):854-861.

55. Pot C, et al. Cutting edge: IL-27 induces the transcription factor c-Maf, cytokine IL-21, and the costimulatory receptor ICOS that coordinately act together to promote differentiation of IL-10-producing Tr1 cells. J Immunol. 2009;183(2):797-801.

56. Saraiva M, Christensen JR, Veldhoen M, Murphy TL, Murphy KM, O'Garra A. Interleukin-10 production by Th 1 cells requires interleukin-12-induced STAT4 transcription factor and ERK MAP kinase activation by high antigen dose. Immunity. 2009; 31(2):209-219.

57. Xu J, et al. c-Maf regulates IL-10 expression during Th17 polarization. I Immunol. 2009; 182(10):6226-6236.

58. Stumhofer JS, et al. Interleukins 27 and 6 induce STAT3-mediated T cell production of interleukin 10. Nat Immunol. 2007;8(12):1363-1371.

59. Gerosa F, et al. Interleukin-12 primes human CD4 and CD8 T cell clones for high production of both interferon- $\gamma$ and interleukin-10. J Exp Med. 1996; 183(6):2559-2569.

60. Perona-Wright G, et al. Systemic but not local infections elicit immunosuppressive IL-10 production by natural killer cells. Cell Host Microbe. 2009; 6(6):503-512

61. Kortylewski M, et al. Regulation of the IL-23 and IL-12 balance by Stat 3 signaling in the tumor microenvironment. Cancer Cell. 2009;15(2):114-123.

62. Fleetwood AJ, Dinh H, Cook AD, Hertzog PJ, Hamilton JA. GM-CSF- and M-CSF-dependent macrophage phenotypes display differential dependence on type I interferon signaling. J Lenkoc Biol. 2009; 86(2):411-421.

63. Gough DJ, et al. Functional crosstalk between type I and II interferon through the regulated expression of STAT1. PLoS Biol. 2010;8(4):e1000361.

64. Heo YJ, et al. IL-10 suppresses Th17 cells and promotes regulatory $\mathrm{T}$ cells in the $\mathrm{CD} 4+\mathrm{T}$ cell population of rheumatoid arthritis patients. Immunol Lett. 2010;127(2):150-156.

65. Wilke CM, et al. Endogenous interleukin-10 constrains Th17 cells in patients with inflammatory bowel disease. J Transl Med. 2011;9:217.

66. Jablonska J, Leschner S, Westphal K, Lienenklaus S, Weiss S. Neutrophils responsive to endogenous IFN-beta regulate tumor angiogenesis and growth in a mouse tumor model. J Clin Invest. 2010; 120(4):1151-1164.

67. Wang L, Pino-Lagos K, de Vries VC, Guleria I, Sayegh MH, Noelle RJ. Programmed death 1 ligand signaling regulates the generation of adaptive Foxp3+CD4+ regulatory T cells. Proc Natl Acad Sci US A. 2008;105(27):9331-9336.

68. Corbett TH, Griswold DP, Roberts BJ, Peckham JC, Schabel FM. Tumor induction relationships in development of transplantable cancers of the colon in mice for chemotherapy assays, with a note on carcinogen structure. Cancer Res. 1975; 35(9):2434-2439.

69. Fox BA, et al. In vitro and in vivo antitumor properties of a T-cell clone generated from murine tumorinfiltrating lymphocytes. J Biol Response Mod. 1990; 9(5):499-511.

70. Nam JS, et al. Transforming growth factor beta subverts the immune system into directly promoting tumor growth through interleukin-17. Cancer Res. 2008;68(10):3915-3923.

71. Punkosdy GA, et al. Regulatory T-cell expansion during chronic viral infection is dependent on endogenous retroviral superantigens. Proc Natl Acad Sci U S A. 2011;108(9):3677-3682.

72. Gough DJ, Messina NL, Clarke CJ, Johnstone RW, Levy DE. Constitutive type I interferon modulates homeostatic balance through tonic signaling. Immunity. 2012;36(2):166-174.

73. Hall JC, Rosen A. Type I interferons: crucial participants in disease amplification in autoimmunity. 
Nat Rev Rheumatol. 2010;6(1):40-49.

74. Pestka S, Krause CD, Walter MR. Interferons, interferon-like cytokines, and their receptors. Immunol Rev. 2004;202:8-32.

75. Byrnes AA, McArthur JC, Karp CL. Interferon- $\beta$ therapy for multiple sclerosis induces reciprocal changes in interleukin-12 and interleukin-10 production. Ann Neurol. 2002;51(2):165-174.

76. Ersoy E, Kus CN, Sener U, Coker I, Zorlu Y. The effects of interferon- $\beta$ on interleukin-10 in multiple sclerosis patients. Eur J Neurol. 2005;12(3):208-211.

77. Dikopoulos N, Bertoletti A, Kroger A, Hauser H, Schirmbeck R, Reimann J. Type I IFN negatively regulates CD8+ T cell responses through IL-10-producing CD4+ T regulatory 1 cells. J Immunol. 2005; 174(1):99-109.

78. Levings MK, Sangregorio R, Galbiati F, Squadrone S, de Waal Malefyt R, Roncarolo MG. IFN- $\alpha$ and IL-10 induce the differentiation of human type $1 \mathrm{~T}$ regulatory cells. J Immunol. 2001;166(9):5530-5539.

79. Teijaro JR, et al. Persistent LCMV infection is controlled by blockade of type I interferon signaling. Science. 2013;340(6129):207-211.

80. Wilson EB, et al. Blockade of chronic type I interferon signaling to control persistent LCMV infection. Science. 2013;340(6129):202-207.

81. Jankovic D, Trinchieri G. IL-10 or not IL-10: that is the question. Nat Immunol. 2007;8(12):1281-1283.

82. Fuertes $M B$, et al. Host type I IFN signals are required for antitumor $\mathrm{CD} 8+\mathrm{T}$ cell responses through CD8 $\{\alpha\}+$ dendritic cells. J Exp Med. 2011; 208(10):2005-2016.

83. Gautier G, et al. A type I interferon autocrine-para- crine loop is involved in Toll-like receptor-induced interleukin-12p70 secretion by dendritic cells. J Exp Med. 2005;201(9):1435-1446.

84. Le Bon A, et al. A role for the transcription factor RelB in IFN- $\alpha$ production and in IFN- $\alpha$-stimulated cross-priming. Eur Immunol. 2006;36(8):2085-2093.

85. Kolumam GA, Thomas S, Thompson LJ, Sprent J, Murali-Krishna K. Type I interferons act directly on CD8 T cells to allow clonal expansion and memory formation in response to viral infection. J Exp Med. 2005;202(5):637-650.

86. Nishibori T, Tanabe Y, Su L, David M. Impaired development of CD4+CD25+ regulatory $\mathrm{T}$ cells in the absence of STAT1: increased susceptibility to autoimmune disease. J Exp Med. 2004;199(1):25-34.

87. Facciabene A, et al. Tumour hypoxia promotes tolerance and angiogenesis via CCL28 and T(reg) cells. Nature. 2011;475(7355):226-230.

88. Liu J, et al. Tumor-associated macrophages recruit CCR6+ regulatory T cells and promote the development of colorectal cancer via enhancing CCL20 production in mice. PLoS One. 2011;6(4):e19495.

89. Stewart CA, Trinchieri G. At 17, in-10's passion need not inflame. Immunity. 2011;34(4):460-462.

90. Tanikawa $\mathrm{T}$, et al. Interleukin-10 ablation promotes tumor development, growth, and metastasis. Cancer Res. 2012;72(2):420-429.

91. Cui W, Liu Y, Weinstein JS, Craft J, Kaech SM. An interleukin-21-interleukin-10-STAT3 pathway is critical for functional maturation of memory CD8+ T cells. Immunity. 2011;35(5):792-805.

92. Sidky YA, Borden EC. Inhibition of angiogenesis by interferons: effects on tumor- and lymphocyte- induced vascular responses. Cancer Res. 1987; 47(19):5155-5161.

93. Zheng H, Qian J, Carbone CJ, Leu NA, Baker DP, Fuchs SY. Vascular endothelial growth factorinduced elimination of the type 1 interferon receptor is required for efficient angiogenesis. Blood. 2011; 118(14):4003-4006.

94. Zhang L, Yuan S, Cheng G, Guo B. Type I IFN promotes IL-10 production from $T$ cells to suppress Th17 cells and Th17-associated autoimmune inflammation. PLoS One. 2011;6(12):e28432.

95. Diamond MS, et al. Type I interferon is selectively required by dendritic cells for immune rejection of tumors. J Exp Med. 2011;208(10):1989-2003.

96. Tan MH, Holyoke ED, Goldrosen MH. Murine colon adenocarcinomas: methods for selective culture in vitro. J Natl Cancer Inst. 1976;56(4):871-873.

97. Fidler IJ. Biological behavior of malignant melanoma cells correlated to their survival in vivo. Cancer Res. 1975;35(1):218-224.

98. Plaksin D, Gelber C, Feldman M, Eisenbach L. Reversal of the metastatic phenotype in Lewis lung carcinoma cells after transfection with syngeneic H-2Kb gene. Proc Natl Acad Sci U S A. 1988; 85(12):4463-4467.

99. Lefrançois L, Lycke N. Isolation of mouse small intestinal intraepithelial lymphocytes, Peyer's patch, and lamina propria cells. Curr Protoc Immunol. 2001; Chapter 3:Unit 3.19

100.Sharov AA, Dudekula DB, Ko MS. A web-based tool for principal component and significance analysis of microarray data. Bioinformatics. 2005; 21(10):2548-2549. 